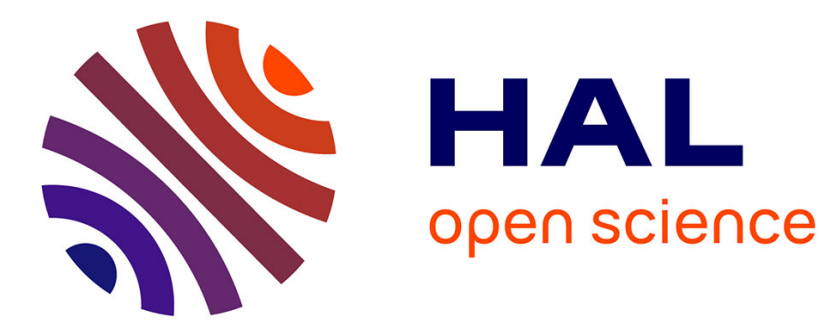

\title{
The DGDD Method for Reduced-Order Modeling of Conservation Laws
}

Sébastien Riffaud, Michel Bergmann, Charbel Farhat, Sebastian Grimberg, Angelo Iollo

\section{- To cite this version:}

Sébastien Riffaud, Michel Bergmann, Charbel Farhat, Sebastian Grimberg, Angelo Iollo. The DGDD Method for Reduced-Order Modeling of Conservation Laws. Journal of Computational Physics, 2021, 437, 10.1016/j.jcp.2021.110336 . hal-03213731v3

\section{HAL Id: hal-03213731 https://hal.inria.fr/hal-03213731v3}

Submitted on 8 Oct 2021

HAL is a multi-disciplinary open access archive for the deposit and dissemination of scientific research documents, whether they are published or not. The documents may come from teaching and research institutions in France or abroad, or from public or private research centers.
L'archive ouverte pluridisciplinaire HAL, est destinée au dépôt et à la diffusion de documents scientifiques de niveau recherche, publiés ou non, émanant des établissements d'enseignement et de recherche français ou étrangers, des laboratoires publics ou privés. 


\title{
The DGDD Method for Reduced-Order Modeling of Conservation Laws
}

\author{
Sébastien Riffaud ${ }^{\mathrm{a}, \mathrm{b}, *}$, Michel Bergmann ${ }^{\mathrm{a}, \mathrm{b}}$, Charbel Farhat ${ }^{\mathrm{c}, \mathrm{d}, \mathrm{e}}$, Sebastian \\ Grimberg ${ }^{\mathrm{c}}$, Angelo Iollo ${ }^{\mathrm{a}, \mathrm{b}}$ \\ ${ }^{a} I M B$, UMR 5251, Univ. Bordeaux, 33400 Talence, France. \\ ${ }^{b}$ INRIA Bordeaux Sud-Ouest, Team MEMPHIS, 33400 Talence, France. \\ ${ }^{c}$ Department of Aeronautics and Astronautics, Stanford University, Stanford, CA 94305, \\ U.S.A. \\ ${ }^{d}$ Department of Mechanical Engineering, Stanford University, Stanford, CA 94305, U.S.A. \\ ${ }^{e}$ Institute for Computational and Mathematical Engineering, Stanford University, Stanford, CA \\ 94305, U.S.A.
}

\begin{abstract}
The discontinuous Galerkin domain decomposition (DGDD) method couples subdomains of high-fidelity polynomial approximation to regions of low-dimensional resolution for the numerical solution of systems of conservation laws. In the lowfidelity regions, the solution is approximated by empirical modes constructed by Proper Orthogonal Decomposition and a reduced-order model is used to predict the solution. The high-dimensional model instead solves the system of conservation laws only in regions where the solution is not amenable to a low-dimensional representation. The coupling between the high-dimensional and the reduced-order models is then performed in a straightforward manner through numerical fluxes at discrete cell boundaries. We show results from application of the proposed method to parametric problems governed by the quasi-1D and 2D compressible Euler equations. In particular, we investigate the prediction of unsteady flows in a converging-diverging nozzle and over a NACA0012 airfoil in presence of shocks. The results demonstrate the stability and the accuracy of the proposed method and the significant reduction of the computational cost with respect to the highdimensional model.
\end{abstract}

Keywords: Reduced-order model, Domain decomposition, Proper Orthogonal Decomposition, Discontinuous Galerkin method, ECSW method

\section{Introduction}

Model order reduction (MOR) is an attractive method to significantly decrease the computational cost associated with numerical solutions of parametric problems

*Corresponding author: sebastien.riffaud@u-bordeaux.fr, sebastien.riffaud@inria.fr 
governed by partial differential equations. It typically consists of a computationally intensive offline training stage where high-fidelity solutions are collected to define a data-driven approximation space and a cheap online simulation stage where the data-driven model is exploited to make new predictions.

Perhaps the most common method for obtaining the reduced approximation space is the Proper Orthogonal Decomposition (POD) [1, 2] which hierarchically rearranges the subspace spanned by high-fidelity solutions according to an energy criterion so that redundant information can be discarded to achieve dimensionality reduction. However, the nature of the problem strongly determines the extent to which one can reduce the dimensionality of the approximation space [3]. As the problem parameters are varied, singular solution features like shocks or compact support phenomena like vortices, shear layers, contact discontinuities, can change their position and shape such that dimensionality reduction is limited. One proposed approach to overcome this limitation is to introduce a mapping applied to the high-fidelity solutions which improves dimensionally reduction [4, 5, 6, 7, 8, 9, Alternatively, in this work we adopt the strategy of employing the reduced-order model (ROM) only in those subdomains where a significant dimensionality reduction can be achieved and employing the high-dimensional model (HDM) elsewhere, as proposed and demonstrated in [3, 10, 11, 12, 13, 14].

The next element in MOR is the formulation of the governing equations for the ROM. The classical approach employs a standard Galerkin projection of the HDM onto the POD subspace. For flow models dominated by advection, special care must be deployed to ensure stability of the resulting ROM. It is well known that standard Galerkin semi-discretization for a linear advection equation is marginally stable in the discrete energy norm and a time-explicit ROM based on such approach is unstable without the introduction of suitable additional numerical diffusion [15]. On the other hand, constructing the ROM based on a discontinuous Galerkin spatial discretization with upwinding of the numerical fluxes is an alternative way to introduce suitable numerical dissipation [16]. The discontinuous Galerkin approach offers the advantage of allowing a modal approximation of the solution and a stable time-explicit discretization. An alternative approach to ensure ROM stability for advection dominated flows consists of minimizing the high-dimensional discrete residual as in the least-squares Petrov-Galerkin projection [17, 18, 19].

In this work, we introduce a discontinuous Galerkin domain decomposition (DGDD) method in which high-dimensional and reduced-order models coexist. Instead of using a global ROM, the domain is spatially partitioned a priori to isolate the subdomains which are anticipated to contain shocks or compact support phenomena. Spatially local ROMs are employed in the subdomains where a significant dimensionality reduction can be achieved while the HDM is used elsewhere. For the coupling, the ROM is based on the discontinuous Galerkin (DG) method [16, 20, 21]. Compared to the standard DG method, the polynomial shape functions are replaced by empirical modes constructed by POD in order to best 
approximate the solution snapshots. In addition, the ROM is equipped with the energy-conserving mesh sampling and weighting (ECSW) hyper-reduction method [22, 23, 24, which provides an empirical quadrature rule enabling the efficient evaluation of the integrals involved in the DG formulation. With this framework, the coupling between the HDM and the local ROMs is performed in a straightforward manner through numerical fluxes at cell boundaries. The accuracy and computational complexity of the resulting method depends on the domain decomposition. If the HDM is used in a large part of the domain, the accuracy of the coupling model can be very high but the resulting model will be computationally expensive to solve. Conversely, if the ROM is sufficient to approximate the solution in most of the domain, this method allows to significantly reduce the computational cost associated with obtaining model solutions in the online stage.

The DGDD method is detailed in the next sections as follows. In Section 2 , we introduce the governing equations and the HDMs employed for their numerical solution. Then, Section 3 presents in details the ROM based on the discontinuous Galerkin method. In Section 4, we describe the domain decomposition and the coupling between the HDM and the ROM. Section 5 demonstrates the accuracy of the proposed method and the significant reduction of the computational cost versus the HDM for three different applications. Finally, Section 6 offers some conclusions.

\section{Governing equations}

Let the parameter domain $\mathcal{D} \in \mathbb{R}^{p}$ be a closed and bounded subset of the Euclidean space $\mathbb{R}^{p}$. Moreover, let the physical domain $\Omega \in \mathbb{R}^{d}$ be a smooth bounded open set with boundary $\partial \Omega$, where $d \in\{1,2\}$ is the space dimension. In this work, we consider parameterized systems of conservation laws of the form

$$
\frac{\partial \mathbf{q}}{\partial t}+\nabla \cdot \mathbf{F}(\mathbf{q})=\mathbf{s}(\mathbf{q})
$$

for $\mathbf{x}=(x, y)^{T} \in \Omega, t \in \mathbb{R}_{+}^{*}, \boldsymbol{\mu} \in \mathcal{D}$, and subject to appropriate initial and boundary conditions. Here $\mathbf{q} \in \mathbb{R}^{N}$ denotes the conservative state variable, $\mathbf{F}=$ $(\mathbf{f}, \mathbf{g})$ denotes the flux and $\mathbf{s}$ denotes the source term. In particular, we will focus on the modeling of inviscid compressible flow with the quasi-1D Euler equations, with

$$
\mathbf{q}=\left(\begin{array}{c}
\rho \\
\rho u \\
E
\end{array}\right), \quad \mathbf{f}=\left(\begin{array}{c}
\rho u \\
\rho u^{2}+p \\
u(E+p)
\end{array}\right), \quad \mathbf{s}=\left(\begin{array}{c}
-\rho u \frac{1}{A} \frac{\mathrm{d} A}{\mathrm{~d} x} \\
-\rho u^{2} \frac{1}{A} \frac{\mathrm{d} A}{\mathrm{~d} x} \\
-u(E+p) \frac{1}{A} \frac{\mathrm{d} A}{\mathrm{~d} x}
\end{array}\right)
$$

and the 2D Euler equations, defined by

$$
\mathbf{q}=\left(\begin{array}{c}
\rho \\
\rho u \\
\rho v \\
E
\end{array}\right), \quad \mathbf{f}=\left(\begin{array}{c}
\rho u \\
\rho u^{2}+p \\
\rho u v \\
u(E+p)
\end{array}\right), \quad \mathbf{g}=\left(\begin{array}{c}
\rho v \\
\rho u v \\
\rho v^{2}+p \\
v(E+p)
\end{array}\right), \quad \mathbf{s}=\left(\begin{array}{l}
0 \\
0 \\
0 \\
0
\end{array}\right)
$$


where $\rho$ is the density, $\mathbf{u}=(u, v)^{T}$ is the velocity, $E$ is the total energy, $A(x) \in$ $\mathcal{C}^{1}(\mathbb{R})$ is a smooth function, for example the cross sectional area of a nozzle, and $p$ is the pressure, given by the equation of state

$$
p=(\gamma-1)\left(E-\rho \frac{\|\mathbf{u}\|^{2}}{2}\right)
$$

with $\gamma$, the specific heat ratio, taken as $\gamma=1.4$ in the following.

In our experiments, the HDM for problems governed by the quasi-1D and 2D Euler equations is constructed using a discontinuous Galerkin method [25, 26] in space and a TVD Runge-Kutta scheme [27] in time.

\section{Reduced-order models based on discontinuous Galerkin method}

In the ROM, each component $q_{i}$ of the solution $\mathbf{q}$ is approximated in space by a small number of basis functions $\Phi_{n}^{i}$ in order to reduce the number of dimension of the solution space:

$$
\forall i \in\{1, \ldots, N\}: \widetilde{q}_{i}(\mathbf{x}, t ; \boldsymbol{\mu})=\bar{q}_{i}(\mathbf{x})+\sum_{n=1}^{M_{i}} a_{n}^{i}(t ; \boldsymbol{\mu}) \Phi_{n}^{i}(\mathbf{x}),
$$

where the offset $\bar{q}_{i}$ and basis functions $\Phi_{n}^{i}$ are built during the offline training stage and the coefficients $a_{n}^{i}$ are computed in the online simulation stage of the ROM.

\subsection{Discontinuous Galerkin method}

In the online stage, the coefficients $a_{n}^{i}$ are determined by the discontinuous Galerkin method. Inserting the approximate solution (4) in the system of conservation laws (1) leads to the residual

$$
\mathcal{R}(\mathbf{x}, t ; \boldsymbol{\mu})=\frac{\partial \widetilde{\mathbf{q}}}{\partial t}+\nabla \cdot \mathbf{F}(\widetilde{\mathbf{q}})-\mathbf{s}(\widetilde{\mathbf{q}})
$$

Projecting this residual onto the basis functions $\Phi_{n}^{i}$, performing an integration by parts on each cell $K \in \Omega$, and replacing the flux by a numerical flux at cell interfaces $\partial K$, we obtain the discontinuous Galerkin spatial discretization of (1)

$$
\frac{\mathrm{d} a_{n}^{i}}{\mathrm{~d} t}=\sum_{K \in \Omega}\left(\int_{K} \mathbf{F}_{i}(\widetilde{\mathbf{q}}) \cdot \nabla \Phi_{n}^{i}+s_{i}(\widetilde{\mathbf{q}}) \Phi_{n}^{i} \mathrm{~d} \mathbf{x}-\int_{\partial K} \widehat{F}_{i}\left(\widetilde{\mathbf{q}}^{-}, \widetilde{\mathbf{q}}^{+}, \mathbf{n}\right) \Phi_{n}^{i} \mathrm{~d} \boldsymbol{\sigma}\right),
$$

where the orthonormality of the basis functions has been used. Here, $\mathbf{n}$ denotes the outward unit normal, $\widehat{\mathbf{F}}_{i}$ denotes the numerical flux, i.e. $\widehat{\mathbf{F}}_{i}=\widehat{\mathbf{F}}_{i}\left(\widetilde{\mathbf{q}}^{-}, \widetilde{\mathbf{q}}^{+}, \mathbf{n}\right)$ with $\widetilde{\mathbf{q}}^{-}$and $\widetilde{\mathbf{q}}^{+}$, the negative and positive trace, respectively, and $\widetilde{\mathbf{q}}^{+}=\mathbf{q}^{b c}$ at the boundary $\partial \Omega$ with $\mathbf{q}^{b c}$ the boundary conditions. Notably, the additional surface integrals provided by the DG formulation are responsible for enforcing in a weak 
sense the boundary conditions and allow to introduce numerical diffusion/dissipation through the numerical flux. To have a consistent scheme, the numerical flux must be consistent, that is

$$
\widehat{\mathbf{F}}_{i}(\widetilde{\mathbf{q}}, \widetilde{\mathbf{q}}, \mathbf{n})=\mathbf{F}_{i}(\widetilde{\mathbf{q}}) \cdot \mathbf{n}
$$

according to Lemma 2.1 in [28]. In this case, the ROM is also consistent.

\subsection{Proper Orthogonal Decomposition}

In the offline stage, the HDM provides a database of $N_{s}$ snapshots of the highfidelity solution collected at different time instances and input parameters. Let $q_{i}^{l}(\mathbf{x})=q_{i}\left(\mathbf{x}, t_{k(l)} ; \boldsymbol{\mu}_{j(l)}\right)$ be the $l^{\text {th }}$ snapshot of the conserved variable $q_{i}$ collected at time instance $t_{k(l)}$ and input parameter $\boldsymbol{\mu}_{j(l)}$. The offset is defined as the mean of the snapshots over time and parameter space:

$$
\bar{q}_{i}(\mathbf{x})=\frac{\sum_{l=1}^{N_{s}} q_{i}^{l}(\mathbf{x})}{N_{s}} .
$$

The basis functions $\Phi_{n}^{i}$ are then computed by POD to yield the best representation of the solution snapshots. More precisely, the subspace spanned by the basis functions $\Phi_{n}^{i}$ is the subspace of rank $M_{i}$ minimizing, in the least-squares sense, the difference between the snapshots and their projections $\mathcal{P}\left[q_{i}^{l}\right]$ onto this subspace. That is, the basis functions $\Phi_{n}^{i}$ are the solution to the minimization problem

$$
\begin{cases}\underset{\Phi_{1}^{i}, \ldots, \Phi_{M_{i}}^{i}}{\operatorname{minimize}} & \sum_{l=1}^{N_{s}} \int_{\Omega}\left(q_{i}^{l}(\mathbf{x})-\mathcal{P}\left[q_{i}^{l}\right](\mathbf{x})\right)^{2} \mathrm{~d} \mathbf{x} \\ \text { subject to } & \int_{\Omega} \Phi_{n}^{i}(\mathbf{x}) \Phi_{m}^{i}(\mathbf{x}) \mathrm{d} \mathbf{x}=\delta_{n, m} \quad \forall n, m \in\left\{1, \ldots, M_{i}\right\},\end{cases}
$$

where $\mathcal{P}\left[q_{i}^{l}\right](\mathbf{x})=\bar{q}_{i}(\mathbf{x})+\sum_{n=1}^{M_{i}} \int_{\Omega}\left(q_{i}^{l}(\mathbf{y})-\bar{q}_{i}(\mathbf{y})\right) \Phi_{n}^{i}(\mathbf{y}) \mathrm{d} \mathbf{y} \Phi_{n}^{i}(\mathbf{x})$. When the snapshots are stored in the matrix

$$
\mathbf{S}^{i}=\left(\begin{array}{cccc}
\stackrel{*}{q}_{i}^{1}\left(\mathbf{x}_{1}\right) & \stackrel{*}{q}_{i}^{2}\left(\mathbf{x}_{1}\right) & \cdots & \stackrel{*}{q}_{i}^{N_{s}}\left(\mathbf{x}_{1}\right) \\
\stackrel{*}{q}_{i}^{1}\left(\mathbf{x}_{2}\right) & \stackrel{*}{q}_{i}^{2}\left(\mathbf{x}_{2}\right) & \cdots & \stackrel{*}{q}_{i}^{N_{s}}\left(\mathbf{x}_{2}\right) \\
\vdots & \vdots & \ddots & \vdots \\
\stackrel{*}{q}_{i}^{*}\left(\mathbf{x}_{N_{\mathbf{x}}}\right) & \stackrel{*}{q}_{i}^{2}\left(\mathbf{x}_{N_{\mathbf{x}}}\right) & \cdots & \stackrel{*}{q}_{i}^{N_{s}}\left(\mathbf{x}_{N_{\mathbf{x}}}\right)
\end{array}\right) \in \mathbb{R}^{N_{\mathbf{x}} \times N_{s}}
$$

with $\stackrel{*}{q}_{i}^{l}(\mathbf{x})=q_{i}^{l}(\mathbf{x})-\bar{q}_{i}(\mathbf{x})$, the basis functions are stored in matrix

$$
\boldsymbol{\Phi}^{i}=\left(\begin{array}{cccc}
\Phi_{1}^{i}\left(\mathbf{x}_{1}\right) & \Phi_{2}^{i}\left(\mathbf{x}_{1}\right) & \cdots & \Phi_{M_{i}}^{i}\left(\mathbf{x}_{1}\right) \\
\Phi_{1}^{i}\left(\mathbf{x}_{2}\right) & \Phi_{2}^{i}\left(\mathbf{x}_{2}\right) & \cdots & \Phi_{M_{i}}^{i}\left(\mathbf{x}_{2}\right) \\
\vdots & \vdots & \ddots & \vdots \\
\Phi_{1}^{i}\left(\mathbf{x}_{N_{\mathbf{x}}}\right) & \Phi_{2}^{i}\left(\mathbf{x}_{N_{\mathbf{x}}}\right) & \cdots & \Phi_{M_{i}}^{i}\left(\mathbf{x}_{N_{\mathbf{x}}}\right)
\end{array}\right) \in \mathbb{R}^{N_{\mathbf{x}} \times M_{i}}
$$


and the quadrature rule is represented by the symmetric positive definite matrix $\mathbf{W} \in \mathbb{R}^{N_{\mathbf{x}} \times N_{\mathbf{x}}}$, the problem $(6)$ can be written in matrix form as

$$
\left\{\begin{array}{lc}
\underset{\boldsymbol{\Phi}^{i}}{\operatorname{minimize}} & \left\|\mathbf{S}^{i}-\boldsymbol{\Phi}^{i}\left(\boldsymbol{\Phi}^{i}\right)^{T} \mathbf{W} \mathbf{S}^{i}\right\|_{F_{W}}^{2} \\
\text { subject to } & \left(\boldsymbol{\Phi}^{i}\right)^{T} \mathbf{W} \boldsymbol{\Phi}^{i}=\mathbf{I}
\end{array}\right.
$$

where $\|\mathbf{A}\|_{F_{W}}^{2}=\operatorname{Tr}\left(\mathbf{A}^{T} \mathbf{W A}\right)$ is the Frobenius norm associated to the scalar product defined by W. By defining $\widehat{\mathbf{S}}^{i}=\left(\mathbf{W}^{\frac{1}{2}}\right)^{T} \mathbf{S}^{i}$, the basis functions are given by the Eckart-Young theorem [29]:

$$
\Phi^{i}=\left(\mathbf{W}^{\frac{1}{2}}\right)^{-T} \mathbf{U}_{M_{i}}
$$

where $\widehat{\mathbf{S}}^{i} \approx \mathbf{U}_{M_{i}} \boldsymbol{\Sigma}_{M_{i}} \mathbf{V}_{M_{i}}^{T}$ is the truncated SVD of $\widehat{\mathbf{S}}^{i}$ and $\mathbf{W}=\mathbf{W}^{\frac{1}{2}}\left(\mathbf{W}^{\frac{1}{2}}\right)^{T}$ is the Cholesky decomposition of $\mathbf{W}$. In the discontinuous Galerkin formulation (5), the derivatives of the basis functions $\nabla \Phi_{n}^{i}$ are also required. As the basis functions are a linear combination of the snapshots, they are derived analytically to obtain

$$
\frac{\partial \boldsymbol{\Phi}^{i}}{\partial x}=\frac{\partial \mathbf{S}^{i}}{\partial x} \mathbf{V}_{M_{i}} \boldsymbol{\Sigma}_{M_{i}}^{-1} \quad \text { and } \quad \frac{\partial \boldsymbol{\Phi}^{i}}{\partial y}=\frac{\partial \mathbf{S}^{i}}{\partial y} \mathbf{V}_{M_{i}} \boldsymbol{\Sigma}_{M_{i}}^{-1}
$$

\subsection{Hyper-reduction}

In order to evaluate the volume integral in system (5), we have to compute the integrand

$$
H_{i, n}^{v}[\widetilde{\mathbf{q}}]=\mathbf{F}_{i}(\widetilde{\mathbf{q}}) \cdot \nabla \Phi_{n}^{i}+s_{i}(\widetilde{\mathbf{q}}) \Phi_{n}^{i}
$$

at each point $\mathbf{x} \in \bigcup_{K \in \Omega} K$, which is in general prohibitively computationally expensive. To resolve this issue, the integrands that are polynomial with respect to $\widetilde{\mathbf{q}}$ (for example $f_{1}=\rho u$ in (2)) are pre-computed in an exact manner since they depend only on the coefficients $a_{n}^{i}$ during the online stage. Alternatively, the ECSW method is employed for the evaluation of the non-polynomial integrands (for example $f_{2}=\rho u^{2}+p$ and $f_{3}=u(E+p)$ in (2) $)$ where the pre-computation-based approach is not applicable. In this method, the integrands are computed online at only a few points $\widetilde{\mathbf{x}} \in \bigcup_{K \in \Omega} K$, and the volume integrals are approximated as

$$
\int_{K \in \Omega} H_{i, n}^{v}[\widetilde{\mathbf{q}}](\mathbf{x}) \mathrm{d} \mathbf{x} \approx \sum_{l=1}^{L_{v}} \widetilde{\omega}_{l}^{v} H_{i, n}^{v}[\widetilde{\mathbf{q}}]\left(\widetilde{\mathbf{x}}_{l}\right)
$$

where $\widetilde{\omega}_{l}^{v}>0$ is the weight associated to the point $\widetilde{\mathbf{x}}_{l}$ and $L_{v} \ll N_{\mathbf{x}}$. The quadrature points and weights are determined simultaneously during the offline stage to 
best approximate the exact volume integral:

$$
\begin{aligned}
& \left(\begin{array}{ccc}
H_{i, n}^{v}\left[\mathbf{q}^{1}\right]\left(\mathbf{x}_{1}\right) & \cdots & H_{i, n}^{v}\left[\mathbf{q}^{1}\right]\left(\mathbf{x}_{N_{\mathbf{x}}}\right) \\
\vdots & & \vdots \\
H_{i, n}^{v}\left[\mathbf{q}^{N_{s}}\right]\left(\mathbf{x}_{1}\right) & \cdots & H_{i, n}^{v}\left[\mathbf{q}^{N_{s}}\right]\left(\mathbf{x}_{N_{\mathbf{x}}}\right)
\end{array}\right)\left(\begin{array}{c}
\omega_{1}^{v} \\
\vdots \\
\omega_{N_{\mathbf{x}}}^{v}
\end{array}\right) \approx\left(\begin{array}{c}
\int_{\bigcup_{K \in \Omega} K} H_{i, n}^{v}\left[\mathbf{q}^{1}\right](\mathbf{x}) \mathrm{d} \mathbf{x} \\
\vdots \\
\int_{\bigcup_{K \in \Omega} K} H_{i, n}^{v}\left[\mathbf{q}^{N_{s}}\right](\mathbf{x}) \mathrm{d} \mathbf{x}
\end{array}\right), \\
& \begin{array}{ccc}
\| & \| & \| \\
\mathbf{G}_{i, n} & \boldsymbol{\omega} & \mathbf{d}_{i, n}
\end{array}
\end{aligned}
$$

where $\mathbf{q}^{l}$ is the $l^{\text {th }}$ snapshot of the conserved variables collected during the offline stage. Combining the contributions of all the integrands $H_{i, n}^{v}$ leads to the formulation

$$
\begin{aligned}
& \left(\begin{array}{c}
\mathbf{G}_{1,1} \\
\mathbf{G}_{1,2} \\
\vdots \\
\mathbf{G}_{2,1} \\
\vdots \\
\mathbf{G}_{N, M_{N}}
\end{array}\right)\left(\begin{array}{c}
\omega_{1}^{v} \\
\vdots \\
\omega_{N_{\mathbf{x}}}^{v}
\end{array}\right) \approx\left(\begin{array}{c}
\mathbf{d}_{1,1} \\
\mathbf{d}_{1,2} \\
\vdots \\
\mathbf{d}_{2,1} \\
\vdots \\
\mathbf{d}_{N, M_{N}}
\end{array}\right) . \\
& \begin{array}{lll}
\| & \| & \| \\
\mathbf{G} & \boldsymbol{\omega} & \mathrm{d}
\end{array}
\end{aligned}
$$

Defining $\Upsilon=\left\{\boldsymbol{\omega} \in \mathbb{R}_{+}^{N_{\mathbf{x}}}:\|\mathbf{G} \boldsymbol{\omega}-\mathbf{d}\|_{2} \leqslant \epsilon\|\mathbf{d}\|_{\mathbf{2}}\right\}, \boldsymbol{\omega}$ is the solution of the minimization problem

$$
\min _{\boldsymbol{\omega} \in \Upsilon}\|\boldsymbol{\omega}\|_{0}
$$

where $\|\cdot\|_{0}$ is the $\ell_{0}$ pseudo-norm. Unfortunately, the problem (8) is NP-hard and in the ECSW method, it is replaced by the non-negative least-squares problem

$$
\min _{\boldsymbol{\omega} \geqslant 0}\|\mathbf{G} \boldsymbol{\omega}-\mathbf{d}\|_{2}^{2}
$$

which is solved by the algorithm described in [30]. This algorithm promotes sparsity in the solution and terminates when the stopping criterion $\|\mathbf{G} \boldsymbol{\omega}-\mathbf{d}\|_{2} \leqslant \epsilon\|\mathbf{d}\|_{2}$ is satisfied for a given level of hyper-reduction accuracy $\epsilon$. The weights $\widetilde{\omega}_{l}$ are finally given by keeping only the nonzero components of the solution of problem (9), and the points $\widetilde{\mathbf{x}}_{l}$ are the points associated to these weights $\widetilde{\omega}_{l}^{v}$. The discontinuous Galerkin ROM (5) then becomes

$$
\frac{\mathrm{d} a_{n}^{i}}{\mathrm{~d} t}=\sum_{l=1}^{L_{v}} \widetilde{w}_{l}^{v} H_{i, n}^{v}[\widetilde{\mathbf{q}}]\left(\widetilde{\mathbf{x}}_{l}\right)-\sum_{K \in \Omega} \int_{\partial K} \widehat{\mathbf{F}}_{i}\left(\widetilde{\mathbf{q}}^{-}, \widetilde{\mathbf{q}}^{+}, \mathbf{n}\right) \Phi_{n}^{i} \mathrm{~d} \boldsymbol{\sigma} .
$$

In the same way, the ECSW method is also employed for the evaluation of the surface integrals by defining

$$
H_{i, n}^{s}[\widetilde{\mathbf{q}}]=\widehat{F}_{i}\left(\widetilde{\mathbf{q}}^{-}, \widetilde{\mathbf{q}}^{+}, \mathbf{n}\right) \Phi_{n}^{i}
$$


for $\boldsymbol{\sigma} \in \bigcup_{K \in \Omega} \partial K$. Finally, the hyper-reduced discontinuous Galerkin ROM is

$$
\frac{\mathrm{d} a_{n}^{i}}{\mathrm{~d} t}=\sum_{l=1}^{L_{v}} \widetilde{\omega}_{l}^{v} H_{i, n}^{v}[\widetilde{\mathbf{q}}]\left(\widetilde{\mathbf{x}}_{l}\right)-\sum_{l=1}^{L_{s}} \widetilde{\omega}_{l}^{s} H_{i, n}^{s}[\widetilde{\mathbf{q}}]\left(\widetilde{\boldsymbol{\sigma}}_{l}\right) .
$$

\subsection{Time discretization}

For the examples in this work, the ROM is discretized in time by the same explicit TVD Runge-Kutta scheme used in the HDM. Writing system (10) as

$$
\frac{\mathrm{d} \mathbf{a}}{\mathrm{d} t}=\mathcal{L}(\mathbf{a}, t ; \boldsymbol{\mu})
$$

where $\mathbf{a}=\left(a_{1}^{1}, a_{2}^{1}, \ldots, a_{1}^{2}, \ldots, a_{M_{N}}^{N}\right)$, the second-order scheme reads

$$
\begin{aligned}
\mathbf{a}^{(1)} & =\mathbf{a}\left(t_{k} ; \boldsymbol{\mu}\right)+\Delta t \mathcal{L}\left(\mathbf{a}\left(t_{k} ; \boldsymbol{\mu}\right), t_{k} ; \boldsymbol{\mu}\right), \\
\mathbf{a}\left(t_{k+1} ; \boldsymbol{\mu}\right) & =\frac{1}{2} a\left(t_{k} ; \boldsymbol{\mu}\right)+\frac{1}{2} \mathbf{a}^{(1)}+\frac{\Delta t}{2} \mathcal{L}\left(\mathbf{a}^{(1)}, t_{k+1} ; \boldsymbol{\mu}\right),
\end{aligned}
$$

and the third-order scheme reads

$$
\begin{aligned}
\mathbf{a}^{(1)} & =\mathbf{a}\left(t_{k} ; \boldsymbol{\mu}\right)+\Delta t \mathcal{L}\left(\mathbf{a}\left(t_{k} ; \boldsymbol{\mu}\right), t_{k} ; \boldsymbol{\mu}\right), \\
\mathbf{a}^{(2)} & =\frac{3}{4} \mathbf{a}\left(t_{k} ; \boldsymbol{\mu}\right)+\frac{1}{4} \mathbf{a}^{(1)}+\frac{\Delta t}{4} \mathcal{L}\left(\mathbf{a}^{(1)}, t_{k+1} ; \boldsymbol{\mu}\right), \\
\mathbf{a}\left(t_{k+1} ; \boldsymbol{\mu}\right) & =\frac{1}{3} \mathbf{a}\left(t_{k} ; \boldsymbol{\mu}\right)+\frac{2}{3} \mathbf{a}^{(2)}+\frac{2 \Delta t}{3} \mathcal{L}\left(\mathbf{a}^{(2)}, t_{k+\frac{1}{2}} ; \boldsymbol{\mu}\right) .
\end{aligned}
$$

The initial solution $\mathbf{a}\left(t_{0} ; \boldsymbol{\mu}\right)$ is given by the orthogonal projection of the initial condition $\mathbf{q}^{0}(\mathbf{x} ; \boldsymbol{\mu})$ onto the approximation subspace.

\section{Discontinuous Galerkin domain decomposition method}

Given the ROM based on the DG method developed in the previous section, the domain decomposition is applied in a straightforward manner since the coupling between the HDM and the ROM is performed though the numerical flux.

\subsection{Domain decomposition}

In the HDM, the domain is divided into micro-cells $K_{j}$ as illustrated by Figure 1. In the standard global MOR approach, these micro-cells are generally agglomerated into a single macro-cell $\Omega$. Thanks to the ROM developed in Section 3, we formulate a spatially local approach for the case of several non-overlapping microand macro-cells as illustrated in Figure 1. The HDM is used in the micro-cells $K_{j}$ while we employ the ROM in the macro-cells $\Omega_{j}$. The domain is decomposed into smooth and non-smooth regions in order to isolate singular solution features 
like shock waves. For simplicity, the partitioning is based in this work on a priori knowledge of the solution, and we anticipate the subdomains representable via POD.
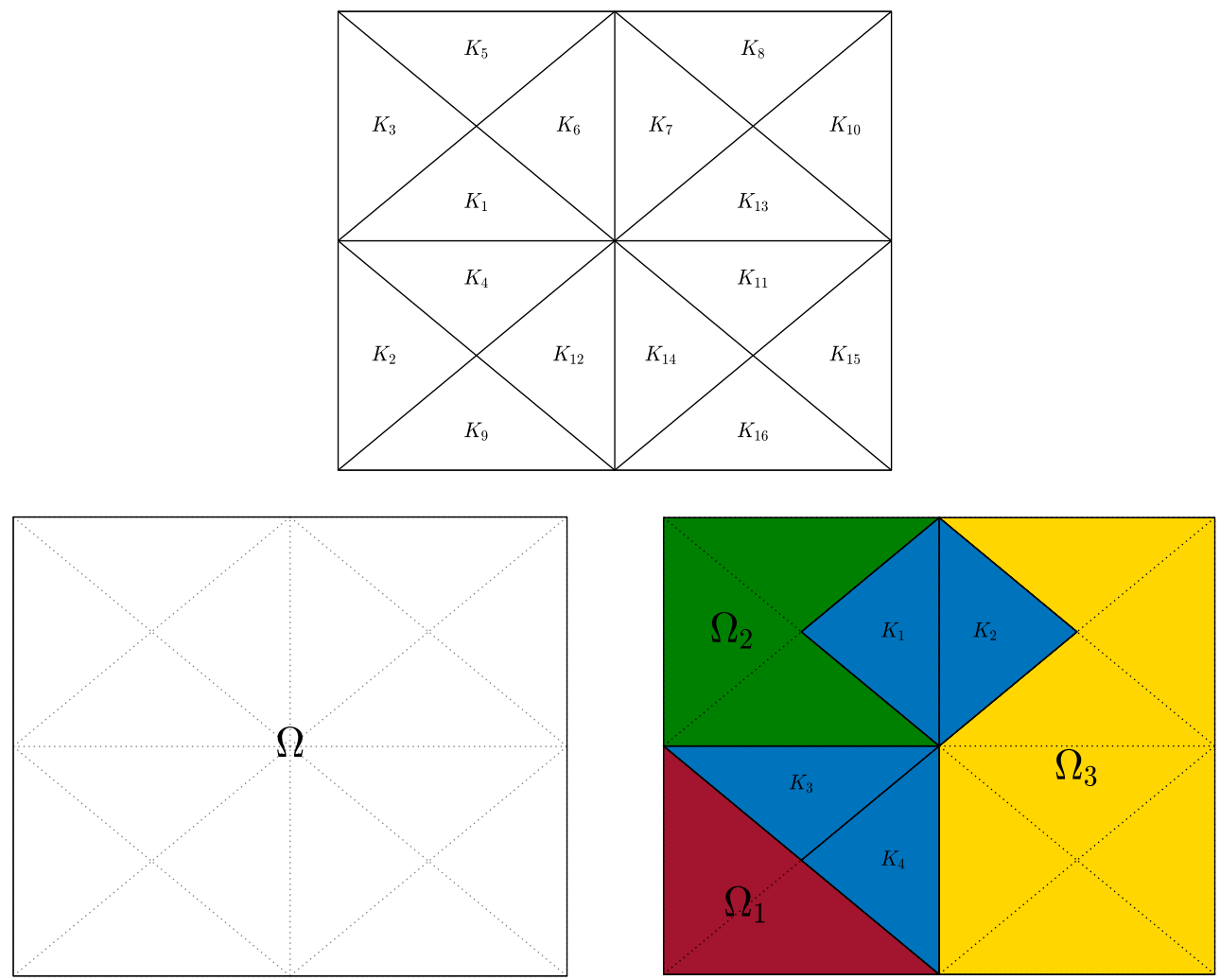

Figure 1: Top: example of mesh employed in the HDM containing 16 micro-cells $K_{j}$. Bottom: examples of domain decomposition. On the left, the domain is agglomerated into a single macrocell. On the right, the domain is divided into 4 micro-cells and 3 macro-cells.

\subsection{Coupling between the HDM and the ROM}

The restriction of the approximate solution to each macro-cell $\Omega_{j}$ is written as

$$
\forall \mathbf{x} \in \Omega_{j}: \widetilde{q}_{i}(\mathbf{x}, t ; \boldsymbol{\mu})=\bar{q}_{i, j}(\mathbf{x})+\sum_{n=1}^{M_{i, j}} a_{n}^{i, j}(t ; \boldsymbol{\mu}) \Phi_{n}^{i, j}(\mathbf{x}),
$$

where we proceed exactly as described in Section 3 for the offline and online stages. The discontinuous Galerkin ROM (10) now becomes

$$
\frac{\mathrm{d} a_{n}^{i, j}}{\mathrm{~d} t}=\sum_{l=1}^{L_{v}^{j}} \widetilde{\omega}_{j, l}^{v} H_{i, j, n}^{v}[\widetilde{\mathbf{q}}]\left(\widetilde{\mathbf{x}}_{j, l}\right)-\sum_{l=1}^{L_{s}^{j}} \widetilde{\omega}_{j, l}^{s} H_{i, j, n}^{s}[\widetilde{\mathbf{q}}]\left(\widetilde{\boldsymbol{\sigma}}_{j, l}\right),
$$

where $H_{i, j, n}^{v}[\widetilde{\mathbf{q}}]=\mathbf{F}_{i}(\widetilde{\mathbf{q}}) \cdot \nabla \Phi_{n}^{i, j}+s_{i}(\widetilde{\mathbf{q}}) \Phi_{n}^{i, j}$ for $\widetilde{\mathbf{x}}_{j, l} \in \bigcup_{K \in \Omega_{j}} K$ and $H_{i, j, n}^{s}\left[\widetilde{\mathbf{q}}_{h}\right]=$ $\widehat{F}_{i}\left(\widetilde{\mathbf{q}}_{h}^{-}, \widetilde{\mathbf{q}}_{h}^{+}, \mathbf{n}\right) \Phi_{n}^{i, j}$ for $\widetilde{\boldsymbol{\sigma}}_{j, l} \in \bigcup_{K \in \Omega_{j}} \partial K$. In this way, the global solution is recov- 
ered by linking the local solutions at the interface between micro- and macro-cells through the numerical flux.

\section{Applications}

In this section, the performance of the DGDD method is evaluated for three applications based on the quasi-1D and 2D Euler equations. In each case, the accuracy of the ROM with respect to the HDM is evaluated using the relative space-time approximation error in the predicted Mach number:

$$
\text { Error }=\sqrt{\frac{\int_{0}^{t_{\max }} \int_{\Omega}\left\|\mathbf{M}_{h d m}-\mathbf{M}_{\text {rom }}\right\|_{2}^{2} \mathrm{~d} \mathbf{x} \mathrm{d} t}{\int_{0}^{t_{\max }} \int_{\Omega}\left\|\mathbf{M}_{h d m}\right\|_{2}^{2} \mathrm{~d} \mathbf{x} \mathrm{d} t}},
$$

where $\mathbf{M}=\mathbf{u} / c$ denotes the Mach number and $c=\sqrt{\gamma p / \rho}$ denotes the speed of sound. Furthermore, the computational speedup of the ROM with respect to the HDM is evaluated in each case in order to quantify the reduction in computational cost provided by ROMs based on the proposed DGDD method.

\subsection{Reproduction of an isentropic vortex}

The first application seeks to validate the DGDD method on a reproductive test case where the online ROM solution is obtained at the same parameter point used for training in the offline stage. We consider an isentropic vortex for $\mathbf{x} \in$ $[0,12] \times[-2.5,2.5]$ and $t \in[0,7]$. The initial and boundary conditions are supplied by the exact solution of the $2 \mathrm{D}$ Euler equations (3):

$$
\left\{\begin{array}{l}
\rho=\left(1-\left(\frac{\gamma-1}{16 \gamma \pi^{2}}\right) \beta^{2} e^{2\left(1-r^{2}\right)}\right)^{\frac{1}{\gamma-1}} \\
u=1-\beta e^{1-r^{2} \frac{y-y_{0}}{2 \pi}} \\
v=\beta e^{1-r^{2} \frac{x-t-x_{0}}{2 \pi}} \\
p=\rho^{\gamma}
\end{array}\right.
$$

with $r=\sqrt{\left(x-t-x_{0}\right)^{2}+\left(y-y_{0}\right)^{2}}, x_{0}=2.5, y_{0}=0$ and $\beta=5$.

The HDM is constructed by discretizing the 2D Euler equations (3) using a third-order discontinuous Galerkin method with piecewise quadratic polynomial approximations and the local Lax-Friedrichs flux in space and the third-order TVD Runge-Kutta method detailed in Section 3.4 in time. The domain is discretized using $N_{K}=960$ triangular micro-cells and the time step is $\Delta t=0.01$. We compare the approximate solutions computed using two ROMs: the first one is a global ROM where the micro-cells are agglomerated into a single macro-cell (i.e. no 
domain decomposition), and the second one is a local ROM where the domain is divided randomly into 8 contiguous macro-cells $\Omega_{j}$ shown in Figure 2 .

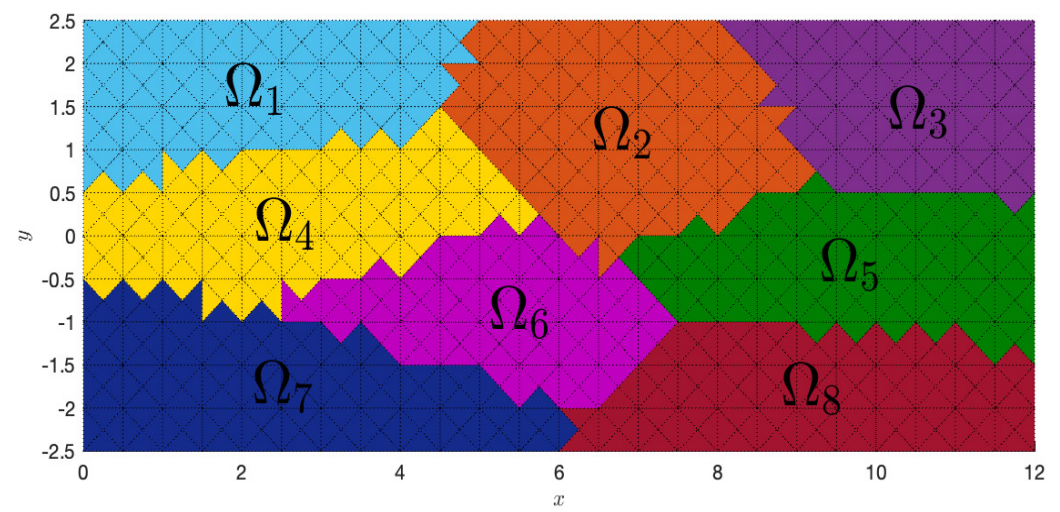

Figure 2: Decomposition of the domain into 8 macro-cells for the isentropic vortex test case.

For this example, no hyper-reduction is used to compare directly the errors introduced due to the different discontinuous Galerkin formulations of the ROM. Snapshots of the HDM solution are collected every time step for the construction of the basis functions. Figure 3 shows snapshots of the Mach number solution computed using the local ROM at different time instances.

Time: 0.00

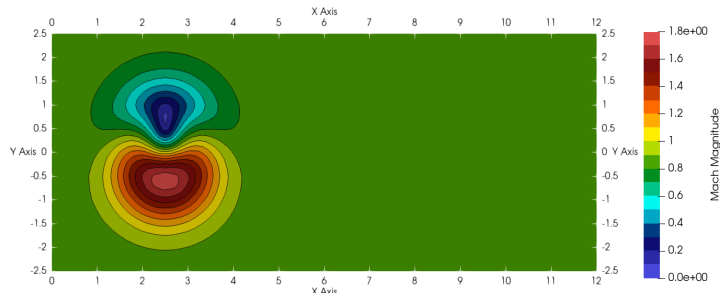

Time: 4.69

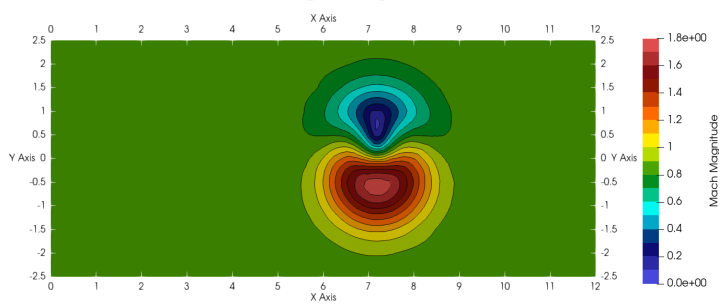

Time: 2.31

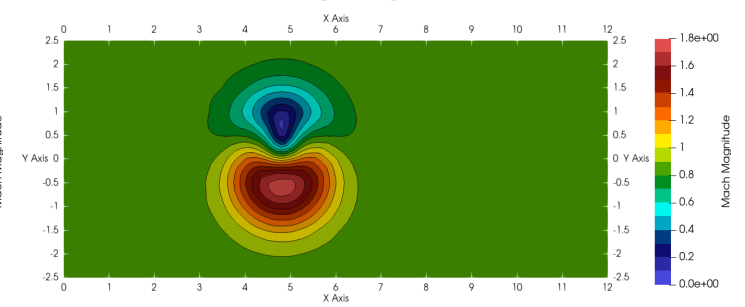

Time: 7.00

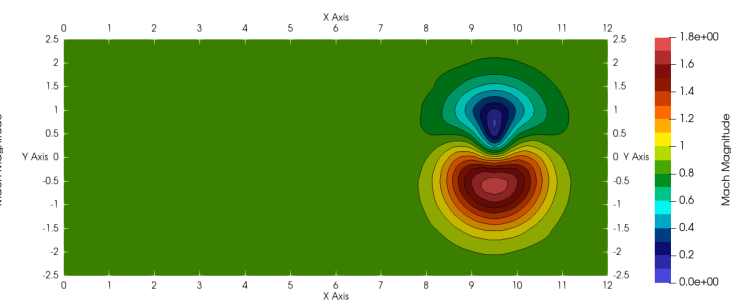

Figure 3: Snapshots of the Mach number solution for the reproduction of the isentropic vortex with 8 macro-cells and $M=15$ basis functions in each macro-cell, as computed using the DGDDbased ROM. The isolines of the corresponding high-fidelity solution are plotted in black.

In Figure 4, we compare the error of the global and local ROMs as a function of the number of basis functions $M$. Here, the number of basis functions is the same for all macro-cells and components of the solution, $\forall i, j: M_{i, j}=M$. The 
error of the global and local ROMs tends to decrease as $M$ increases, even though the convergence is not necessarily monotonic. Moreover, the error of the ROMs is close to the $L^{2}$ projection error of individual state, and the local ROM is more accurate than the global ROM, which validates the proposed DGDD approach.

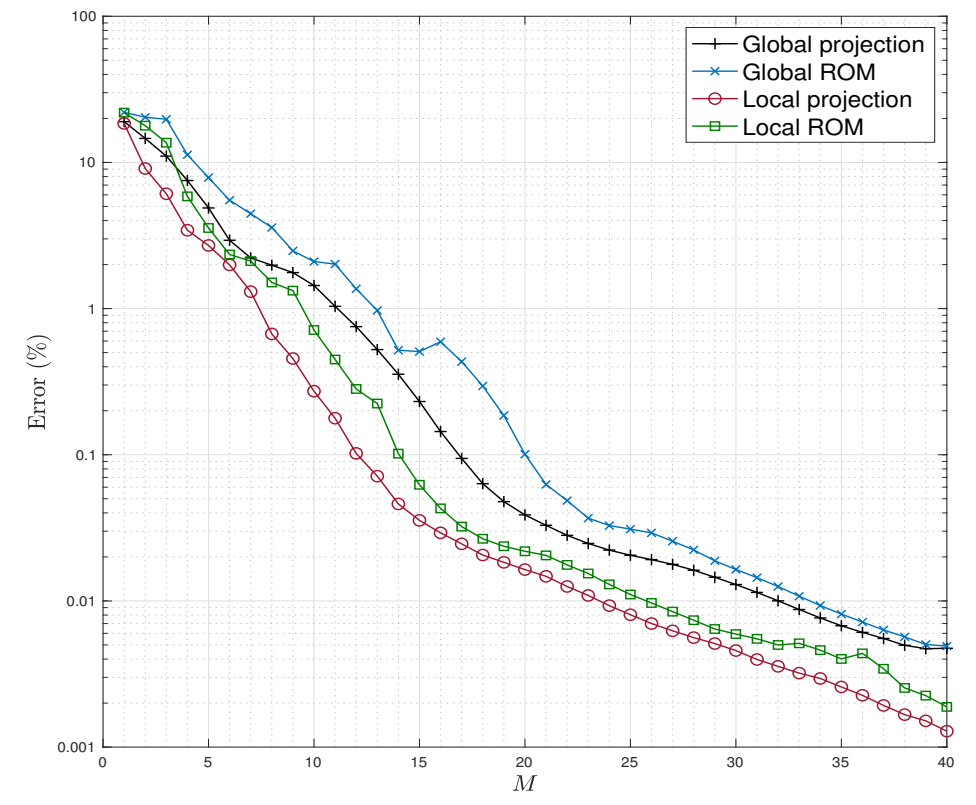

Figure 4: Error of the global and local approaches for the reproduction of an isentropic vortex.

\subsection{Prediction of a transonic flow in a converging-diverging nozzle}

The second application considers the prediction of a transonic flow in a convergingdiverging nozzle for $x \in[0,1]$ and $t \in[0,5]$. The cross sectional area of the nozzle for this application is illustrated in Figure 5.
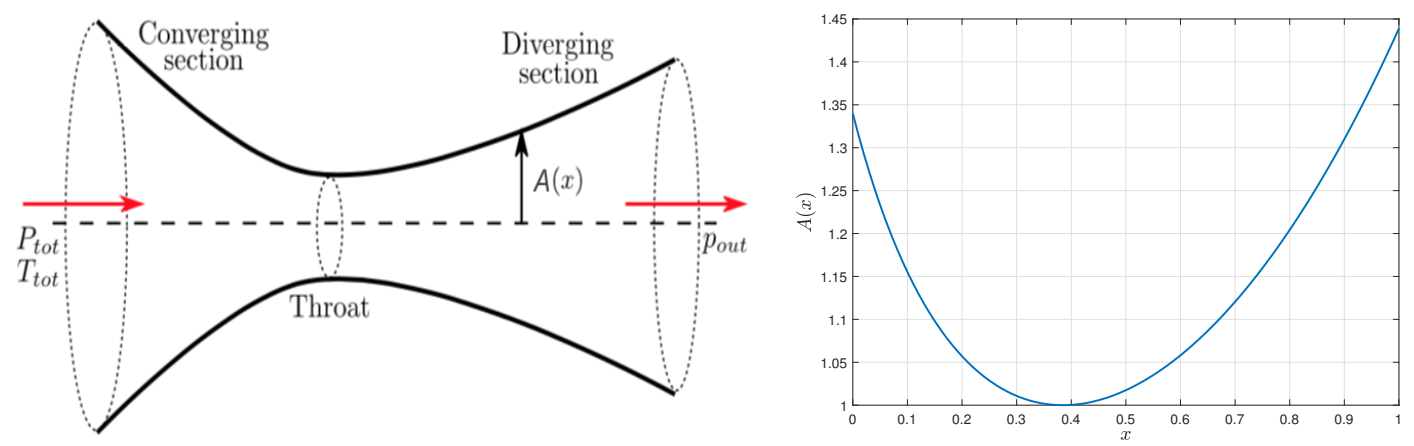

Figure 5: On the left, converging-diverging nozzle [31. On the right, cross sectional area profile $A(x)=\frac{1}{0.5+1.3 x}\left(\frac{2+(\gamma-1)(0.5+1.3 x)^{2}}{1+\gamma}\right)^{\frac{\gamma+1}{2(\gamma-1)}}$.

The steady state solution is determined by the total pressure $P_{\text {tot }}$ and total temperature $T_{\text {tot }}$ at the inlet and by the pressure $p_{\text {out }}$ at the outlet. In this example, 
the total temperature at the inlet is fixed to $T_{t o t}=1$ and the problem parameters $\boldsymbol{\mu}=\left(P_{t o t}, x_{s}\right)$ are the inlet total pressure $P_{t o t}$ and the position of the shock wave $x_{s}$ which is a function of $P_{\text {tot }}$ and $p_{\text {out }}$. We consider the unsteady problem, starting from the initial solution given in Figure 6 and with time-dependent boundary conditions which move the position of the shock wave as follows:

$$
P_{\text {tot }}^{b c}(t ; \boldsymbol{\mu})=\left\{\begin{array}{ll}
1+\frac{P_{t o t}-1}{0.1} t & \text { if } t<0.1 \\
P_{t o t} & \text { else, }
\end{array} \quad x_{s}^{b c}(t ; \boldsymbol{\mu})= \begin{cases}0.7+\frac{x_{s}-0.7}{0.1} t & \text { if } t<0.1 \\
x_{s} & \text { else. }\end{cases}\right.
$$

The quasi-1D Euler equations (2) are discretized using a second-order Discontinuous Galerkin method equipped with the local Lax-Friedrichs flux and the minmod limiter 32] in space and the second-order TVD Runge-Kutta scheme from Section 3.4 in time. The domain is discretized using $N_{K}=500$ micro-cells and the time step is $\Delta t=0.0008$. Domain decomposition is performed from a priori knowledge of the solution by dividing the physical domain into three regions as shown by Figure 6. In non-shocked regions 1 and 3, spatially local ROMs are used and the micro-cells are agglomerated into a single macro-cell for each region. Region 2 consists of 100 micro-cells where the HDM is used in order to accurately capture the moving shock wave.
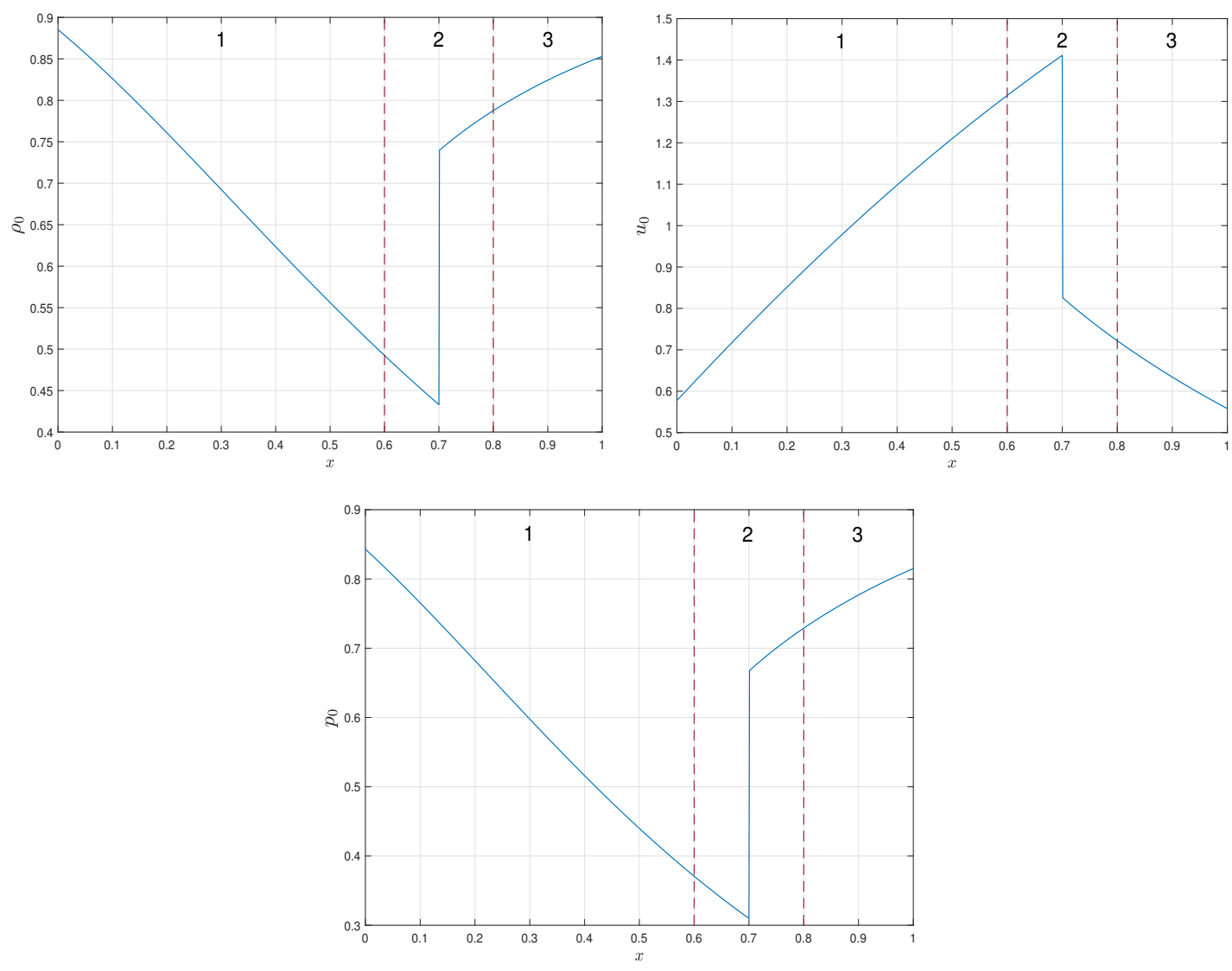

Figure 6: Initial condition corresponding to the steady state solution for $\boldsymbol{\mu}=(1,0.7)$. The domain is divided in 2 macro-cells, denoted by regions 1 and 3, and 100 micro-cells in region 2 . 
Figure 7 illustrates the parameter domain of interest, chosen in order to place the shock wave in the interval $x_{s} \in[0.61,0.79]$. It also shows the sampled training parameter points used to build the snapshot database in the offline stage. Note that the training parameter points corresponding to $\boldsymbol{\mu}=\left(P_{t o t}, 0.7\right)$ have been removed from the original sampling since they correspond to the initial solution and are already in the snapshot database. For each unsteady simulation corresponding to a sampled parameter point, we collect one snapshot every 5 time steps from the HDM simulation.

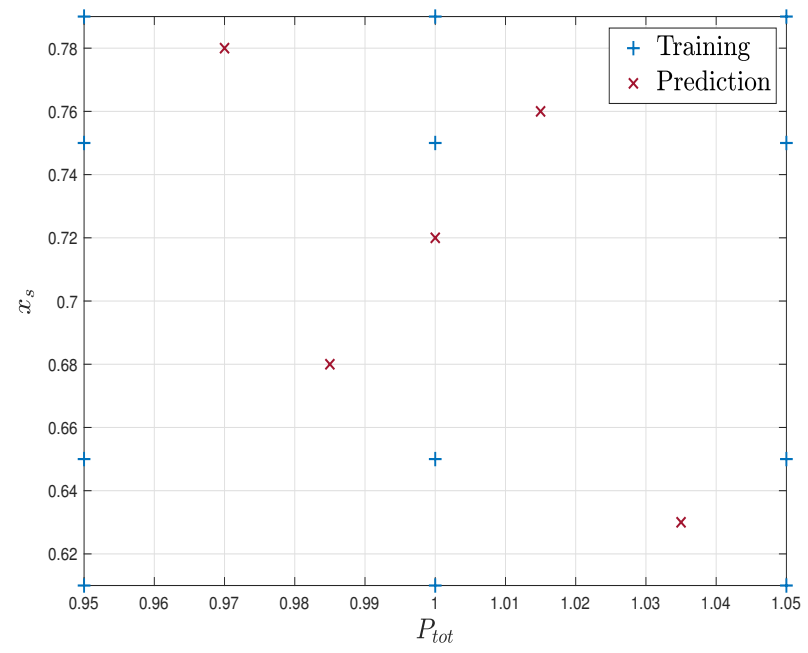

Figure 7: Parameter domain of interest and offline and online sampling for the convergingdiverging nozzle problem.

The singular values of the snapshot matrix for each of the conservative variables are shown in Figure 8. The squared singular values decrease rapidly, and 2 basis functions are sufficient to obtain a relative squared projection error lower than $0.001 \%$ for all variables in each macro-cell.
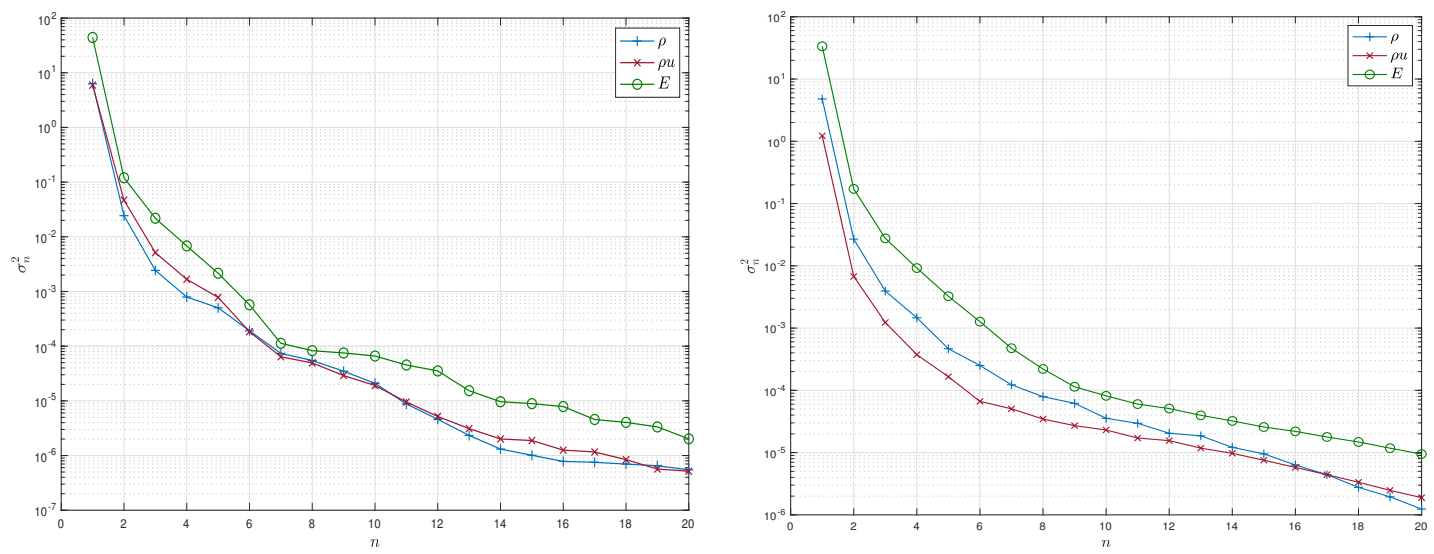

Figure 8: Squared singular values of the snapshot matrix corresponding to region 1 on the left and region 3 on the right for the converging-diverging nozzle problem. 
The hyper-reduction training tolerance for this problem is chosen by a trial and error approach as $\epsilon=10^{-5}$ in regions 1 and 3 which is sufficient to yield an accurate approximation of the integrals in the online stage. In region 1, the ECSW procedure identifies $L_{v}^{1}=23\left(\operatorname{resp} . L_{s}^{1}=9\right)$ points $\widetilde{x}_{l}$ (resp. $\left.\widetilde{\sigma}_{l}\right)$ among the 900 (resp. 301) quadrature points to evaluate the volume (resp. surface) integrals. In region 3, the ECSW procedure identifies 49 (resp. 33) points $\widetilde{x}_{l}$ (resp. $\widetilde{\sigma}_{l}$ ) among the 300 (resp. 101) quadratures points to evaluate the volume (resp. surface) integrals. The reduced mesh delivered by the ECSW method is displayed in Figure 9. Notably, the ECSW procedure identifies more points in region 3, where a wave is moving at the beginning of the simulations (see Figure 10), than in region 1, where the flow solution is more amenable to a low-dimensional representation.

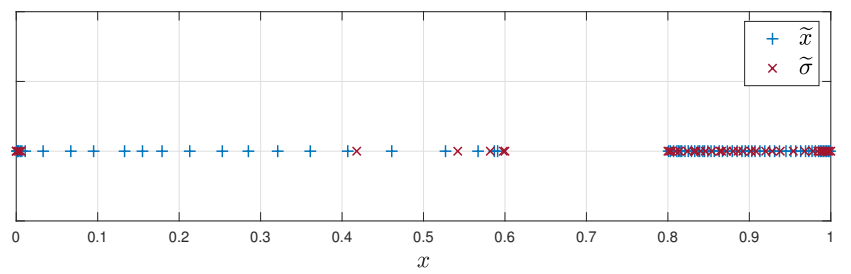

Figure 9: Quadrature points delivered by the ECSW method to approximate the volume (blue) and surface (red) integrals for $M=8$.
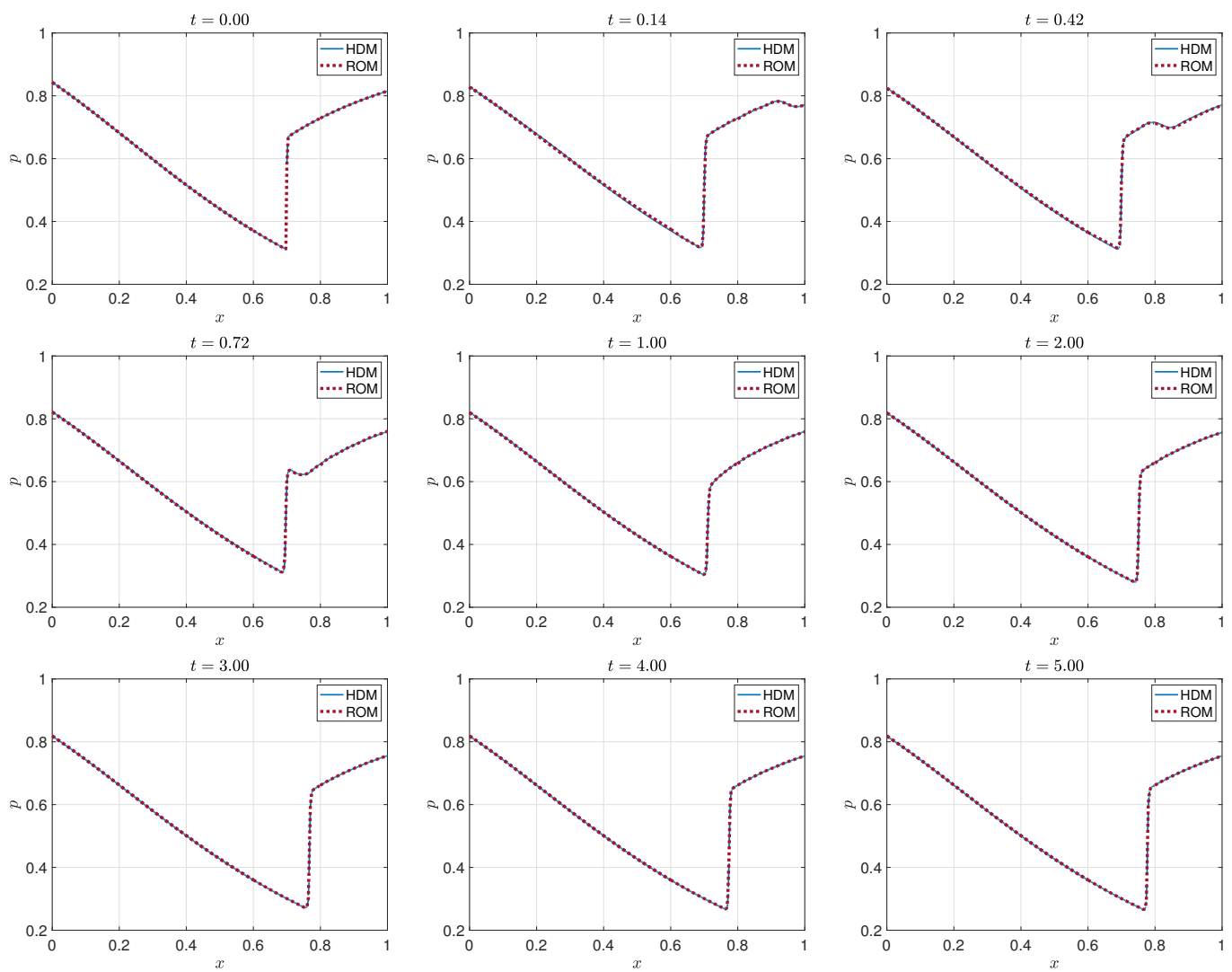

Figure 10: Computed pressure solution snapshots for the prediction test $P_{t o t}=0.97, x_{s}=0.78$ with $M=8$ for the converging-diverging nozzle problem at different time instances. 


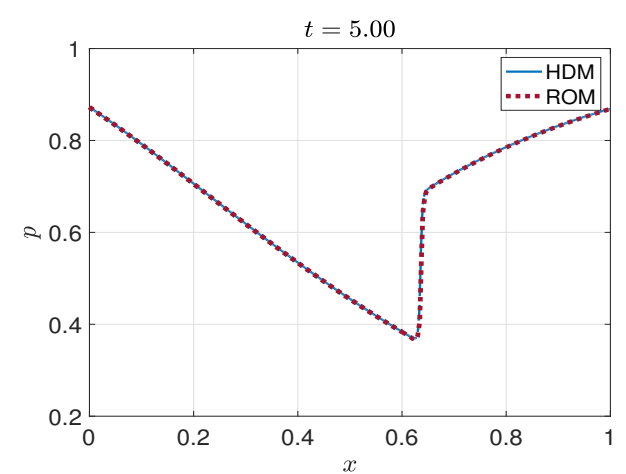

(a) $P_{t o t}=1.035, x_{s}=0.63$

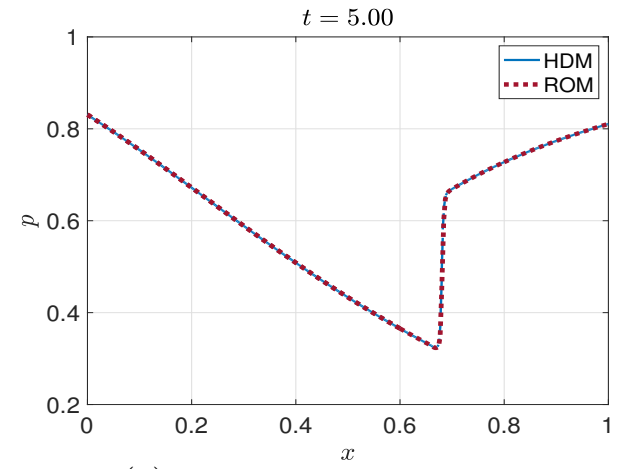

(c) $P_{t o t}=0.985, x_{s}=0.68$

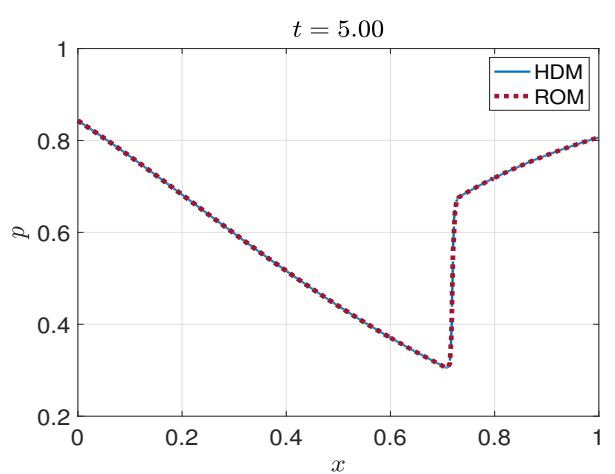

(b) $P_{\text {tot }}=1, x_{s}=0.72$

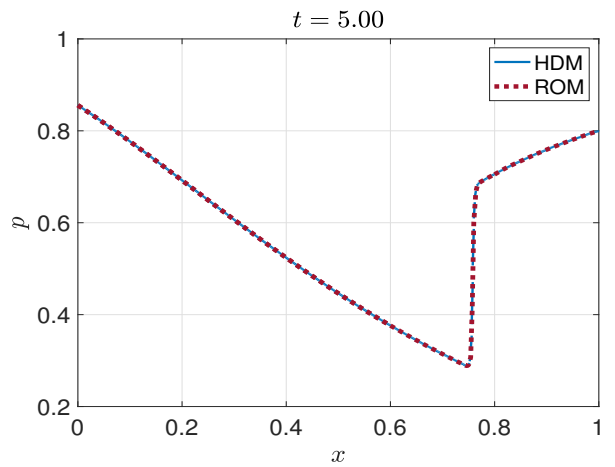

(d) $P_{t o t}=1.015, x_{s}=0.76$

Figure 11: Computed pressure solutions for the prediction tests at steady state with $M=8$ for the converging-diverging nozzle problem.

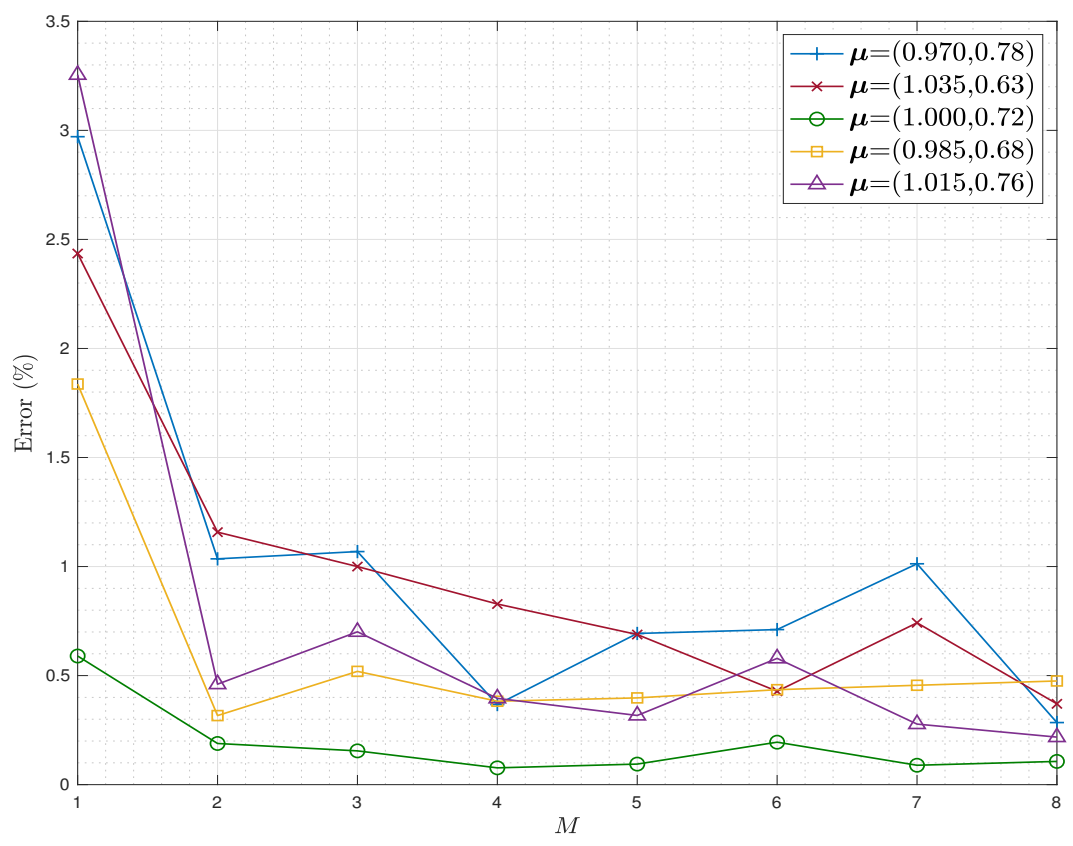

Figure 12: Error of the prediction tests for the converging-diverging nozzle problem as a function of the number of basis functions in region 3 . 
Figures 10 and 11 show the pressure solutions obtained by the DGDD-based ROM for the different parameter prediction tests denoted in Figure 7. In Figure 12 , we compare the error of the prediction tests as a function of the number of basis functions in region 3. The number of basis functions is chosen to be the same for all conservative variables and the number of basis functions in region 1 is fixed to $M=2$. As expected, the error tends to decrease when the number of basis functions $M$ increases and the error is less than $1 \%$ when $M=4$ for all prediction tests. Notably, the error comes mainly from region 2, since a small perturbation of the shock wave position results in a large approximation error. For this small problem, using $M=4$, the computational speedup factor for the solution of the ROM versus the HDM is 3.54. Of the time required for the solution of the DGDDbased ROM, $70.76 \%$ is spent in the computation of the HDM solution in region 2 and the remaining $29.24 \%$ is spent for the local ROMs in regions 1 and 3 .

\subsection{Prediction of a transonic flow over a NACA 0012 airfoil}

For the final application, we consider a 2D transonic flow over a NACA 0012 airfoil. We want to predict the flow solution at parameters $\boldsymbol{\mu}=\left(M_{\infty}, \alpha\right)$ corresponding to different free-stream Mach numbers $M_{\infty}$ and angles of attack $\alpha$. The initial condition is a uniform flow at Mach $M_{\infty}$

$$
\mathbf{x} \in \Omega: \rho^{0}(\mathbf{x} ; \boldsymbol{\mu})=\gamma, u^{0}(\mathbf{x} ; \boldsymbol{\mu})=M_{\infty}, v^{0}(\mathbf{x} ; \boldsymbol{\mu})=0, p^{0}(\mathbf{x} ; \boldsymbol{\mu})=1,
$$

and the unsteady solution is computed for $t \in[0,21]$. Slip boundary conditions are applied at the airfoil surface and the far-field boundary condition is set to be a uniform flow at Mach $M_{\infty}$.

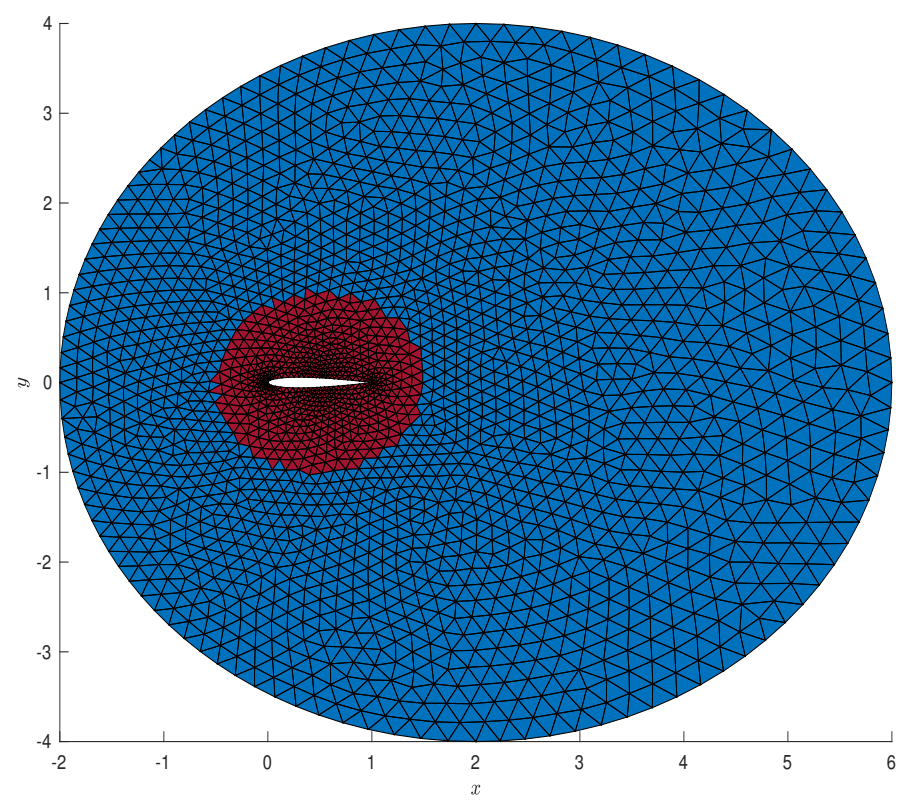

Figure 13: Decomposition of the domain in 1065 micro-cells (red) and one macro-cell (blue) for the transonic NACA airfoil problem. 
The HDM is constructed by discretizing the 2D Euler equations (3) using a second-order discontinuous Galerkin method equipped with the HLL flux [33] and the Barth-Jespersen limiter [34] in space and the second-order TVD Runge-Kutta scheme from Section 3.4 in time. The domain is discretized using $N_{K}=4150$ triangular micro-cells and the time step is $\Delta t=0.003$. As shown in Figure 13 , the domain is divided in two regions: the HDM is employed in the region near the airfoil to accurately capture the moving shock wave, while the ROM is used elsewhere where the solution is amenable to accurate low-dimensional representation in the parameter domain of interest.

In Figure 14, we plot the sampling of the parameter domain of interest for the offline training stage and online prediction stage. For each HDM simulation in the offline stage, one snapshot is collected every 25 time steps. A unique set of basis functions is constructed for each online prediction parameter point. For each prediction point, we use POD on the snapshots corresponding to the four closest training parameter points to the prediction point, defined by the square grid containing the predicted parameter value. For example, the basis functions for the queried parameter point corresponding to $M_{\infty}=0.784$ and $\alpha=0.6$ are computed using the snapshots from the simulations corresponding to $\left(M_{\infty}, \alpha\right) \in$ $\{(0.78,0.5),(0.79,0.5),(0.79,1),(0.78,1))\}$.

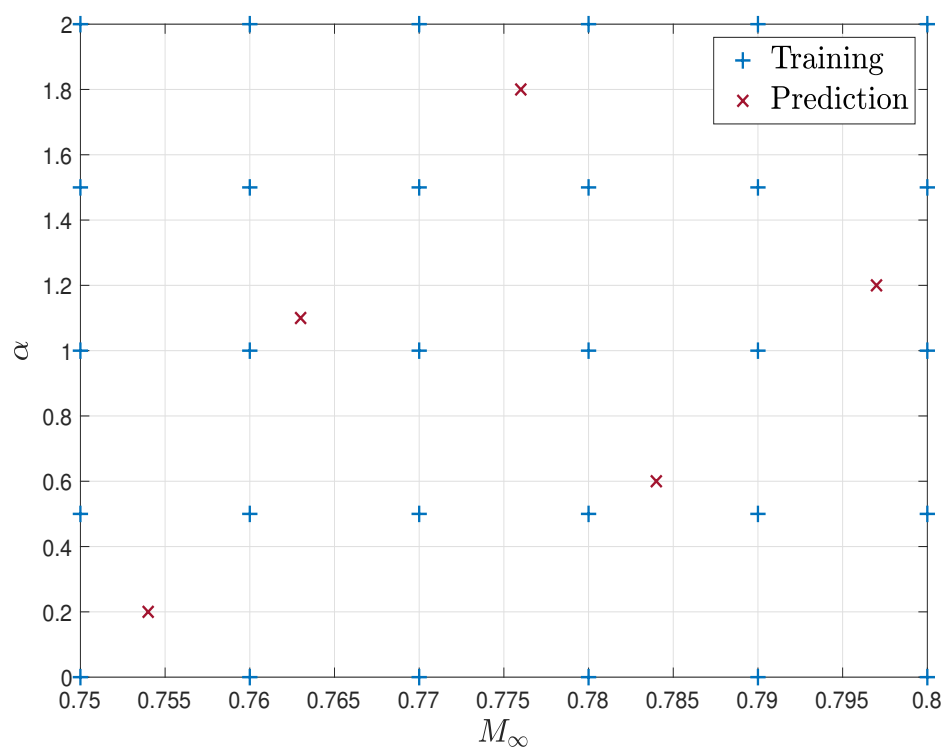

Figure 14: Offline and online parameter domain sampling for the transonic NACA airfoil problem.

Figure 15 plots the decay of the singular values of the snapshot matrix for each of the conservative variables for this example. In this case, 6 basis functions are required to obtain a relative squared projection error of less than $0.001 \%$ for $\rho$, $\rho u$ and $E$. The momentum in the y-direction is close to zero, and the (absolute) squared projection error is below 0.3 with 6 basis functions for $\rho v$. 


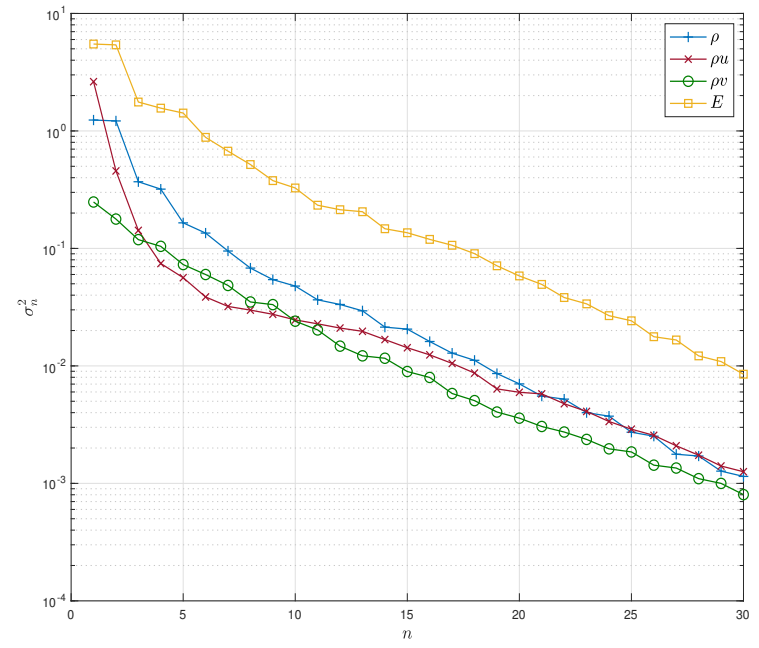

Figure 15: Squared singular values of the snapshot matrix corresponding to the online prediction point $M_{\infty}=0.784$ and $\alpha=0.6$ for the transonic NACA airfoil problem.

Lastly, the hyper-reduction tolerance is chosen by a trial and error approach as $\epsilon=10^{-4}$ which provides for sufficient accuracy in the online stage. For the test corresponding to $\boldsymbol{\mu}=(0.754,0.2)$, the ECSW procedure identifies $L_{v}=403$ (resp. $\left.L_{s}=629\right)$ points $\widetilde{\mathbf{x}}_{l}\left(\right.$ resp. $\widetilde{\boldsymbol{\sigma}}_{l}$ ) among the 9255 (resp. 9314) quadrature points to evaluate the volume (resp. surface) integrals. Figure 16 shows the resulting reduced mesh. The quadrature points are notably located on the left side of the domain and in the airfoil's wake, where compression waves and shocks are propagating before the stationary solution is established. For the other testcases, the result of the ECSW procedure is similar.

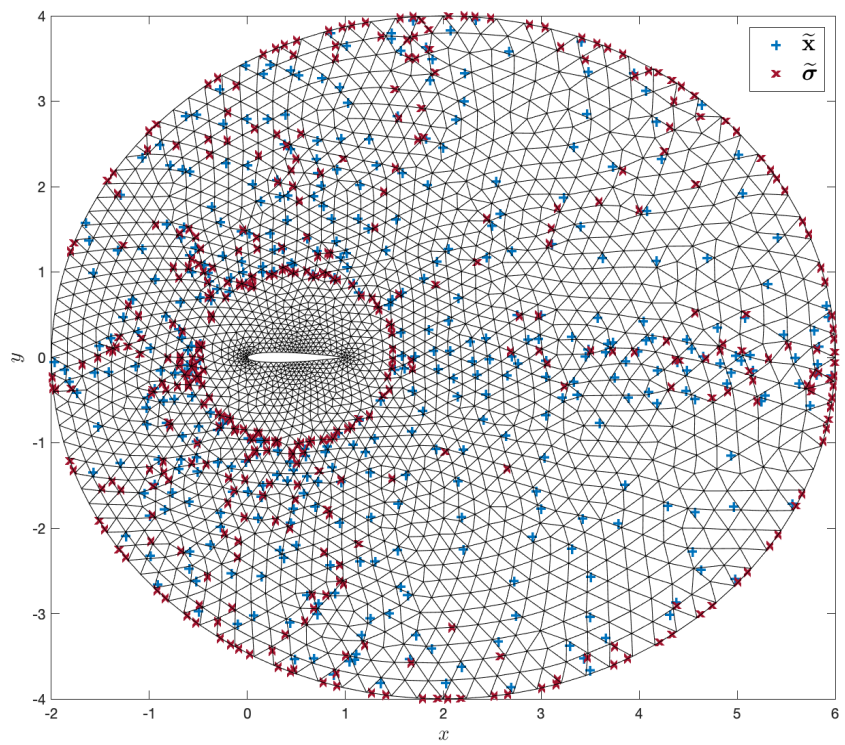

Figure 16: Quadrature points delivered by the ECSW method to approximate the volume (blue) and surface (red) integrals for $M=16$. 
Figures 17 and 18 show snapshots of the computed Mach number using the DGDD-based ROM for the different prediction parameter points and for different times during the time-dependent flow simulations.

In Figure 19, we plot the space-time error depending on the number of basis functions for each of the prediction tests. The number of basis functions is again taken to be the same for all variables. It can be observed that the approximation error for the DGDD-based ROM is low even when using a small number of basis functions. When $M=7$, the prediction error is less than $1 \%$ for all prediction tests. As the number of basis functions $M$ increases, the approximation error decreases slowly which is symptomatic of the slow singular value decay demonstrated in Figure 15 . With $M=7$, the computational speedup factor delivered by the ROM over the HDM simulation is 4.54. Of the time required for the solution of the DGDD-based ROM, 94.41\% comes from the micro-cell solution using the HDM while the remaining $5.59 \%$ comes from the single ROM macro-cell.
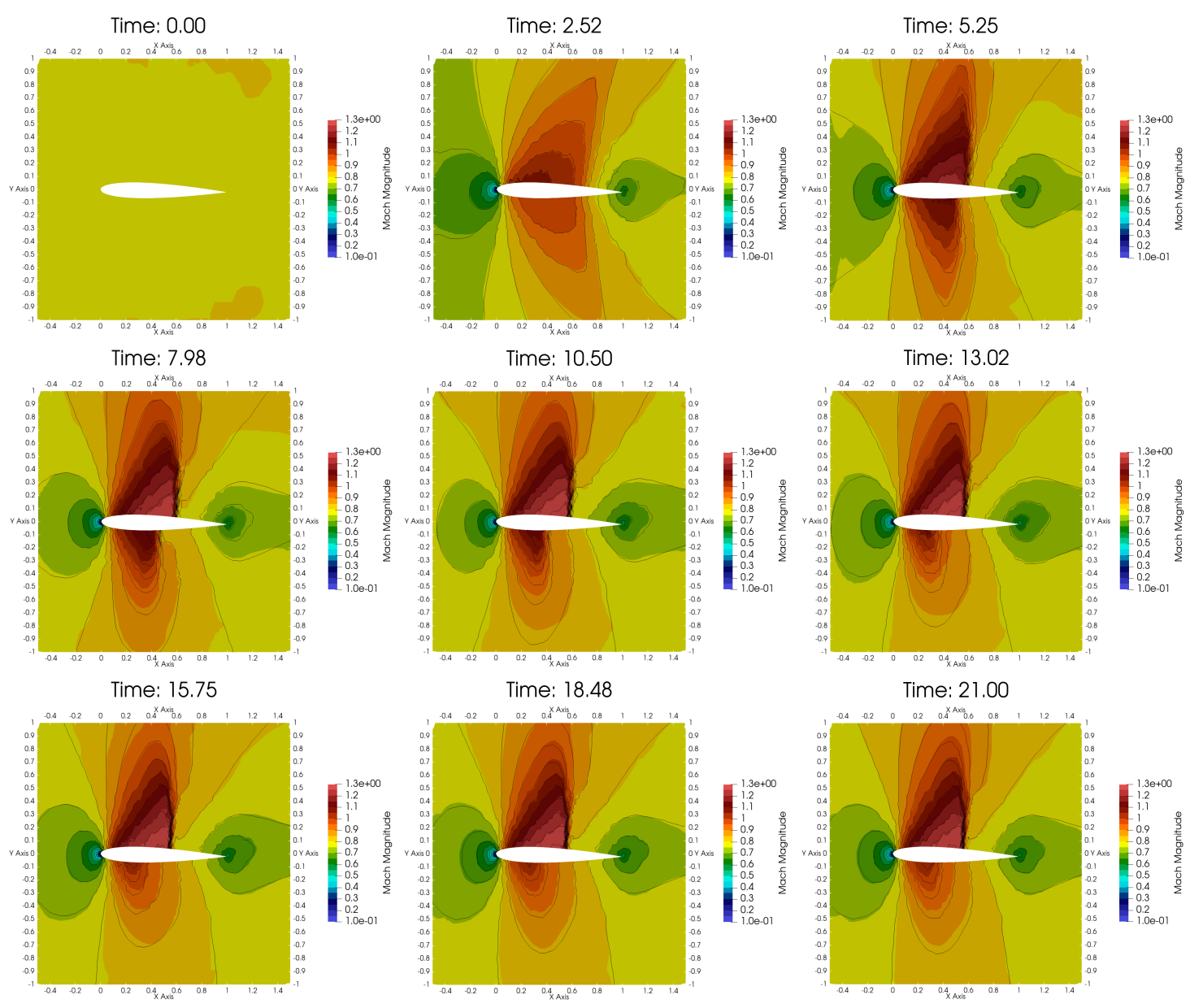

Figure 17: Mach number solution snapshots for the transonic NACA airfoil problem at different time instances computed using the ROM for the prediction test $M_{\infty}=0.797$ and $\alpha=1.2$ with $M=16$. The isolines of the corresponding high-fidelity solution are plotted in black. 
Time: 21.00

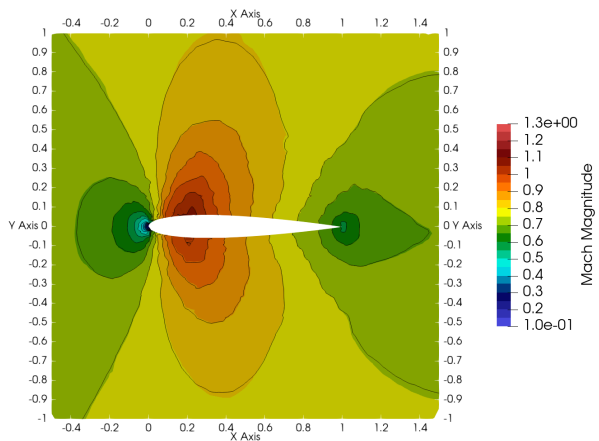

(a) $M_{\infty}=0.754, \alpha=0.2$

Time: 21.00

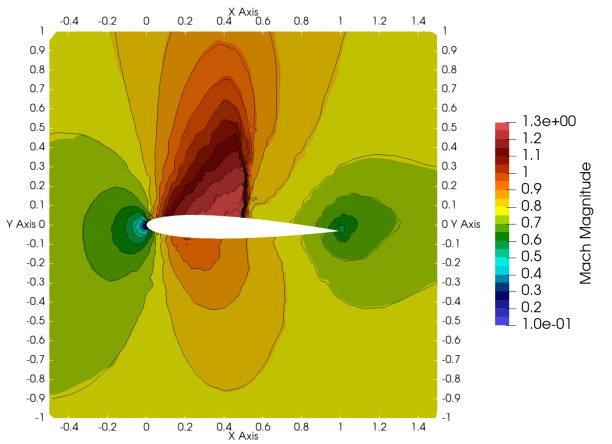

(c) $M_{\infty}=0.776, \alpha=1.8$
Time: 21.00

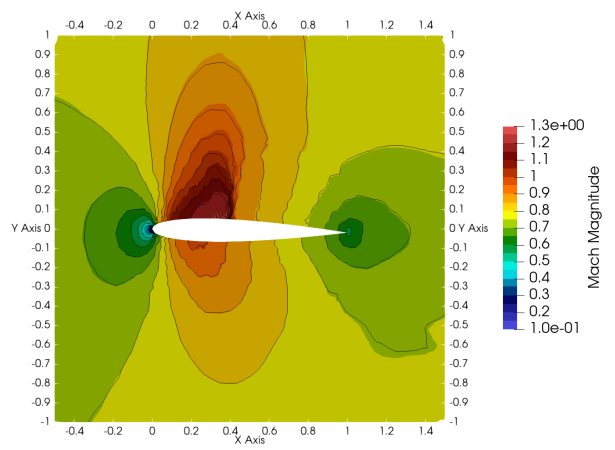

(b) $M_{\infty}=0.763, \alpha=1.1$

Time: 21.00

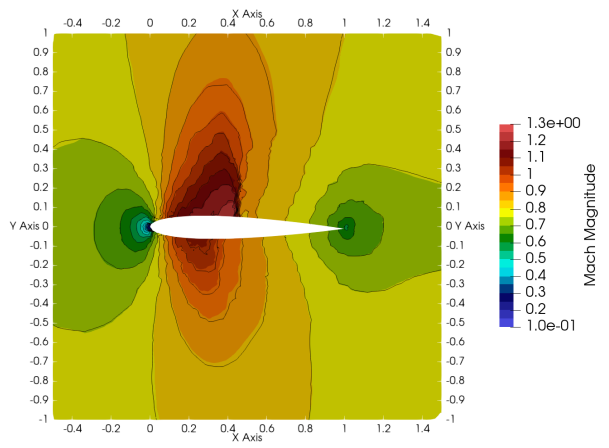

(d) $M_{\infty}=0.784, \alpha=0.6$

Figure 18: Mach number solution snapshots for the transonic NACA airfoil problem at steady state with $M=16$. The isolines of the corresponding high-fidelity solution are plotted in black.

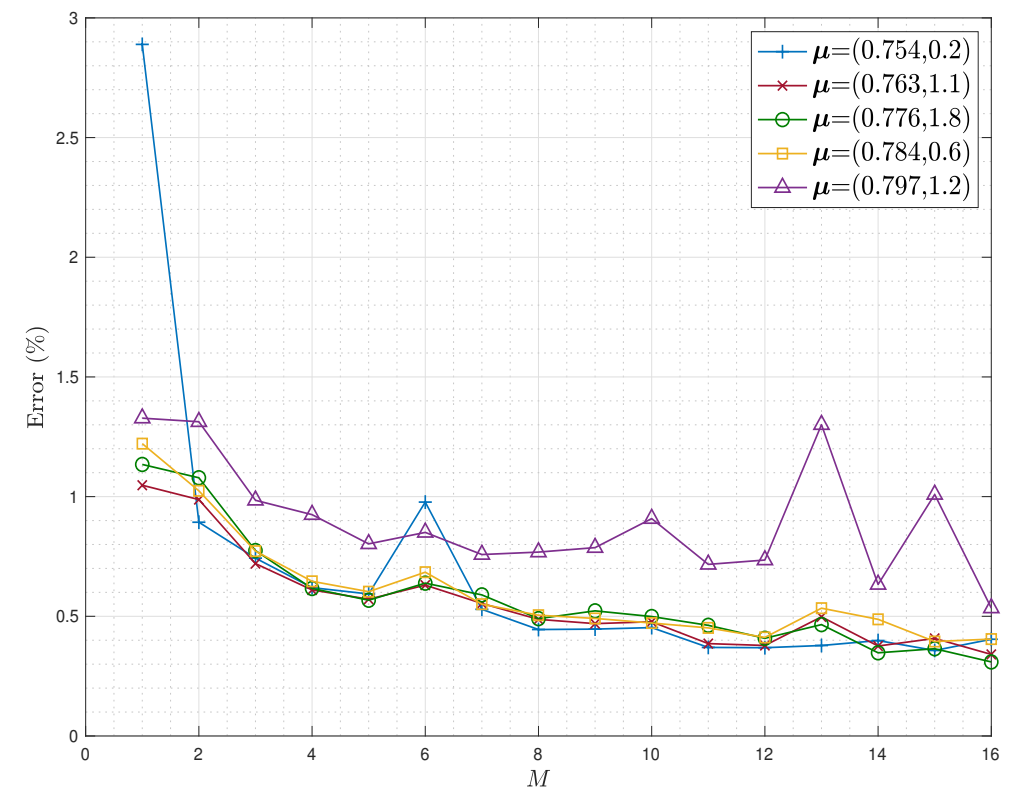

Figure 19: Error of the prediction tests for the transonic NACA airfoil problem as a function of the number of basis functions. 


\section{Conclusions}

In this work, we have presented a DGDD method for systems of conservation laws. In this approach, a ROM approximates the solution in regions where significant dimensionality reduction can be achieved while the HDM is employed elsewhere. The discontinuous Galerkin formulation for the ROM offers a simple way to perform the coupling between the HDM and ROMs.

ROMs based on the proposed DGDD framework have been evaluated for parametric problems governed by the quasi-1D and 2D Euler equations. We have validated the DGDD method on the reproduction of an isentropic vortex. We have then investigated the prediction of unsteady flows in a converging-diverging nozzle and over a NACA 0012 airfoil. The results demonstrate the accuracy of the method, capable of delivering less than $1 \%$ of error over a range of predictive parameter points, and the significant reduction of the required computation time for the ROM simulations versus the associated HDM.

The computational complexity of the DGDD-based ROMs could be further reduced by optimally reducing the number of micro- and macro-cells. To this end, the decomposition of the domain can be made based on an error indicator as in 35] instead of using an a priori decomposition. Another perspective could be to derive some sort of element-wise error model to switch from low-fidelity to high-fidelity. By employing the HDM only when necessary, this approach could reduce the computational cost of the DGDD-based ROMs, while locally increasing the approximation of the solution. Furthermore, this method can be extended to higher order differential equations, including elliptic problems and the NavierStokes equations, by adapting the discontinuous Galerkin method developed in [36, 37, 38, 39, 40, 41] to the ROM approach. Moreover, the method is easily applicable to other modal representations such as the reduced basis method 42 , 43]. These topics will be the subject of future work.

\section{Aknowledgements}

This project has received funding from the European Union's Horizon 2020 research and innovation programme under the Marie Skłodowska-Curie grant agreement No 872442. Experiments presented in this paper were carried out using the PlaFRIM experimental testbed, supported by Inria, CNRS (LABRI and IMB), Université de Bordeaux, Bordeaux INP and Conseil Régional d'Aquitaine (see https://www.plafrim.fr/).

\section{References}

[1] L. Sirovich, Turbulence and the dynamics of coherent structures. I. Coherent structures, Quarterly of applied mathematics 45 (3) (1987) 561-571. 
[2] G. Berkooz, P. Holmes, J. L. Lumley, The proper orthogonal decomposition in the analysis of turbulent flows, Annual review of fluid mechanics 25 (1) (1993) 539-575.

[3] D. Lucia, P. King, M. Oxley, P. Beran, Reduced order modeling for a onedimensional nozzle flow with moving shocks, in: 15th AIAA computational fluid dynamics conference, 2001, p. 2602.

[4] R. Abgrall, D. Amsallem, R. Crisovan, Robust model reduction by $L^{1}$-norm minimization and approximation via dictionaries: application to nonlinear hyperbolic problems, Advanced Modeling and Simulation in Engineering Sciences 3 (1) (2016) 1.

[5] A. Iollo, D. Lombardi, Advection modes by optimal mass transfer, Physical Review E 89 (2) (2014) 022923.

[6] A. Mendible, S. L. Brunton, A. Y. Aravkin, W. Lowrie, J. N. Kutz, Dimensionality Reduction and Reduced Order Modeling for Traveling Wave Physics, arXiv preprint arXiv:1911.00565.

[7] N. J. Nair, M. Balajewicz, Transported snapshot model order reduction approach for parametric, steady-state fluid flows containing parameter dependent shocks, arXiv preprint arXiv:1712.09144.

[8] F. Bernard, A. Iollo, S. Riffaud, Reduced-order model for the BGK equation based on POD and optimal transport, Journal of Computational Physics 373 (2018) 545-570.

[9] M. Nonino, F. Ballarin, G. Rozza, Y. Maday, Overcoming slowly decaying Kolmogorov n-width by transport maps: application to model order reduction of fluid dynamics and fluid-structure interaction problems, arXiv preprint arXiv:1911.06598.

[10] P. LeGresley, J. Alonso, Dynamic domain decomposition and error correction for reduced order models, in: 41st Aerospace Sciences Meeting and Exhibit, 2003, p. 250.

[11] P. A. LeGresley, Application of proper orthogonal decomposition (POD) to design decomposition methods, Stanford University, 2006.

[12] M. Buffoni, H. Telib, A. Iollo, Iterative methods for model reduction by domain decomposition, Computers \& Fluids 38 (6) (2009) 1160-1167.

[13] L. Iapichino, A. Quarteroni, G. Rozza, A reduced basis hybrid method for the coupling of parametrized domains represented by fluidic networks, Computer Methods in Applied Mechanics and Engineering 221 (2012) 63-82. 
[14] L. Iapichino, A. Quarteroni, G. Rozza, Reduced basis method and domain decomposition for elliptic problems in networks and complex parametrized geometries, Computers \& Mathematics with Applications 71 (1) (2016) 408430 .

[15] A. Iollo, S. Lanteri, J.-A. Désidéri, Stability properties of POD-Galerkin approximations for the compressible Navier-Stokes equations, Theoretical and Computational Fluid Dynamics 13 (6) (2000) 377-396.

[16] A. Iollo, A. Dervieux, J.-A. Désidéri, S. Lanteri, Two stable POD-based approximations to the Navier-Stokes equations, Computing and visualization in science 3 (1-2) (2000) 61-66.

[17] K. Carlberg, M. Barone, H. Antil, Galerkin v. least-squares Petrov-Galerkin projection in nonlinear model reduction, Journal of Computational Physics 330 (2017) 693-734.

[18] K. Carlberg, C. Bou-Mosleh, C. Farhat, Efficient non-linear model reduction via a least-squares Petrov-Galerkin projection and compressive tensor approximations, International Journal for Numerical Methods in Engineering 86 (2) (2011) 155-181.

[19] S. Grimberg, C. Farhat, N. Youkilis, On the stability of projection-based model order reduction for convection-dominated laminar and turbulent flows, Journal of Computational Physics 419 (2020) 109681.

[20] P. F. Antonietti, P. Pacciarini, A. Quarteroni, A discontinuous Galerkin reduced basis element method for elliptic problems, ESAIM: Mathematical Modelling and Numerical Analysis 50 (2) (2016) 337-360.

[21] M. Yano, Discontinuous Galerkin reduced basis empirical quadrature procedure for model reduction of parametrized nonlinear conservation laws, Advances in Computational Mathematics 45 (5-6) (2019) 2287-2320.

[22] C. Farhat, P. Avery, T. Chapman, J. Cortial, Dimensional reduction of nonlinear finite element dynamic models with finite rotations and energy-based mesh sampling and weighting for computational efficiency, International Journal for Numerical Methods in Engineering 98 (9) (2014) 625-662.

[23] C. Farhat, T. Chapman, P. Avery, Structure-preserving, stability, and accuracy properties of the energy-conserving sampling and weighting method for the hyper reduction of nonlinear finite element dynamic models, International Journal for Numerical Methods in Engineering 102 (5) (2015) 1077-1110.

[24] S. Grimberg, C. Farhat, R. Tezaur, C. Bou-Mosleh, Mesh sampling and weighting for the hyperreduction of nonlinear Petrov-Galerkin reduced-order 
models with local reduced-order bases, International Journal for Numerical Methods in Engineering 122 (7) (2021) 1846-1874.

[25] B. Cockburn, C.-W. Shu, The Runge-Kutta discontinuous Galerkin method for conservation laws V: multidimensional systems, Journal of Computational Physics 141 (2) (1998) 199-224.

[26] J. S. Hesthaven, T. Warburton, Nodal discontinuous Galerkin methods: algorithms, analysis, and applications, Springer Science \& Business Media, 2007.

[27] C.-W. Shu, S. Osher, Efficient implementation of essentially non-oscillatory shock-capturing schemes, Journal of computational physics 77 (2) (1988) 439471.

[28] S. Gérald, Méthode de Galerkin Discontinue et intégrations explicitesimplicites en temps basées sur un découplage des degrés de liberté. Applications au système des équations de Navier-Stokes., Ph.D. thesis, Université Pierre et Marie Curie - Paris VI (2013).

[29] C. Eckart, G. Young, The approximation of one matrix by another of lower rank, Psychometrika 1 (3) (1936) 211-218.

[30] C. L. Lawson, R. J. Hanson, Solving least squares problems, Vol. 15, Siam, 1995.

[31] E. Abbate, A. Iollo, G. Puppo, An all-speed relaxation scheme for gases and compressible materials, Journal of Computational Physics 351 (2017) 1-24.

[32] P. L. Roe, Characteristic-based schemes for the Euler equations, Annual review of fluid mechanics 18 (1) (1986) 337-365.

[33] A. Harten, P. D. Lax, B. v. Leer, On upstream differencing and Godunov-type schemes for hyperbolic conservation laws, SIAM review 25 (1) (1983) 35-61.

[34] D. Kuzmin, A vertex-based hierarchical slope limiter for p-adaptive discontinuous Galerkin methods, Journal of computational and applied mathematics 233 (12) (2010) 3077-3085.

[35] M. Bergmann, A. Ferrero, A. Iollo, E. Lombardi, A. Scardigli, H. Telib, A zonal Galerkin-free POD model for incompressible flows, Journal of Computational Physics 352 (2018) 301-325.

[36] D. N. Arnold, An interior penalty finite element method with discontinuous elements, SIAM journal on numerical analysis 19 (4) (1982) 742-760.

[37] F. Bassi, S. Rebay, A high-order accurate discontinuous finite element method for the numerical solution of the compressible Navier-Stokes equations, Journal of computational physics 131 (2) (1997) 267-279. 
[38] C. E. Baumann, J. T. Oden, A discontinuous hp finite element method for convection-diffusion problems, Computer Methods in Applied Mechanics and Engineering 175 (3-4) (1999) 311-341.

[39] B. Cockburn, C.-W. Shu, The local discontinuous Galerkin method for timedependent convection-diffusion systems, SIAM Journal on Numerical Analysis 35 (6) (1998) 2440-2463.

[40] J. Peraire, P.-O. Persson, The compact discontinuous Galerkin (CDG) method for elliptic problems, SIAM Journal on Scientific Computing 30 (4) (2008) $1806-1824$.

[41] B. Van Leer, S. Nomura, Discontinuous Galerkin for diffusion, in: 17th AIAA Computational Fluid Dynamics Conference, 2005, p. 5108.

[42] B. Haasdonk, M. Ohlberger, Reduced basis method for finite volume approximations of parametrized linear evolution equations, ESAIM: Mathematical Modelling and Numerical Analysis 42 (2) (2008) 277-302.

[43] G. Rozza, D. B. P. Huynh, A. T. Patera, Reduced basis approximation and a posteriori error estimation for affinely parametrized elliptic coercive partial differential equations, Archives of Computational Methods in Engineering 15 (3) (2008) 1. 